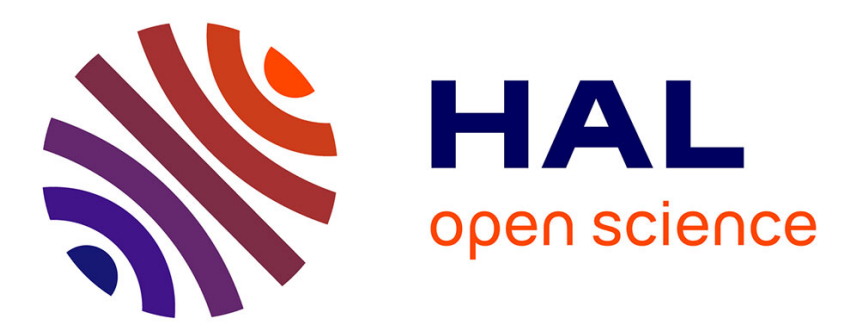

\title{
Influence of the sampling density on the noise level in displacement and strain maps obtained by processing periodic patterns
}

Siyi Qin, Michel Grédiac, Benoît Blaysat, Shaopeng Ma, Frédéric Sur

\section{To cite this version:}

Siyi Qin, Michel Grédiac, Benoît Blaysat, Shaopeng Ma, Frédéric Sur. Influence of the sampling density on the noise level in displacement and strain maps obtained by processing periodic patterns. Measurement - Journal of the International Measurement Confederation (IMEKO), 2021, 173, pp.108570. 10.1016/j.measurement.2020.108570 . hal-02965328

\section{HAL Id: hal-02965328 \\ https://hal.science/hal-02965328}

Submitted on 7 Apr 2021

HAL is a multi-disciplinary open access archive for the deposit and dissemination of scientific research documents, whether they are published or not. The documents may come from teaching and research institutions in France or abroad, or from public or private research centers.
L'archive ouverte pluridisciplinaire $\mathbf{H A L}$, est destinée au dépôt et à la diffusion de documents scientifiques de niveau recherche, publiés ou non, émanant des établissements d'enseignement et de recherche français ou étrangers, des laboratoires publics ou privés. 


\title{
Influence of the sampling density on the noise level in displacement and strain maps obtained by processing periodic patterns
}

\author{
S. Qin ${ }^{1}$, M. Grédiac ${ }^{2}$, B. Blaysat ${ }^{2}$, S. Ma ${ }^{3}$, F. Sur ${ }^{4}$ \\ ${ }^{1}$ Beijing Institute of Technology, Beijing, China \\ ${ }^{2}$ Institut Pascal, UMR 6602, Université Clermont-Auvergne, CNRS, SIGMA Clermont, \\ Clermont-Ferrand, France \\ ${ }^{3}$ Department of Engineering Mechanics, School of Naval Architecture, Ocean and Civil Engineering, \\ Jiao Tong University, Shanghai, China \\ ${ }^{4}$ LORIA, UMR 7503, Université de Lorraine, CNRS, Inria, Nancy, France
}

\begin{abstract}
The aim of this study is to investigate the influence of the sampling density of periodic patterns such as checkerboards or 2D grids on the noise level observed in displacement and strain maps extracted from such images. A spectral method named Localized Spectrum Analysis (LSA) is used to process the images. It is shown that this parameter influences this noise level. This influence is quantified, and a power law is proposed to model it, with a value of the power identified with the measurements. It is also confirmed that checkerboards give a lower noise level than $2 \mathrm{D}$ grids, with an improvement which is also quantified, and which increases as the sampling density increases.
\end{abstract}

Keywords: Checkerboard, Digital image correlation, Grid method, Heteroscedastic noise, Localized Spectrum Analysis, Metrology, Optimal pattern, Uncertainty quantification, Windowed Fourier transform

This is the author-manuscript version of

S. Qin, M. Grédiac, B. Blaysat, S. Ma, F. Sur. Influence of the sampling density on the noise level in displacement and strain maps obtained by processing periodic patterns. Measurement, vol. 173, p. 108570, Elsevier, 2021.

DOI: $10.1016 / j . m e a s u r e m e n t .2020 .108570$ 


\section{Introduction}

Studying the metrological performance of full-field measurement techniques is the topic of many recent papers, in particular concerning Digital Image Correlation (DIC) thanks to the collaborative work carried out within the framework of the DIC-Challenge [1]. Displacement and strain maps are now widely used to reveal material heterogeneities $[2,3]$, to track crack propagation in engineering materials $[4,5]$, or to identify material properties [6, 7], among other applications. In this context, determining reliable maps is a chief issue. The metrological performance is generally defined in terms of spatial resolution (denoted here by $d$ ), measurement resolution (displacement resolution $\sigma_{u}$ and strain resolution $\sigma_{\varepsilon}$ ) and bias $\lambda$ [8]. These quantities are thoroughly defined in Appendix A but in short: the spatial resolution reflects the ability of the measuring technique to distinguish close features in displacement and strain maps, the measurement resolution represents the noise level in those maps, and the bias the systematic error impairing displacement and strain values returned by the technique.

For a given technique, users often want to estimate $d, \sigma_{u}, \sigma_{\varepsilon}$ and $\lambda$, but the problem is that these quantities depend on various parameters, some of them being extrinsic to the measuring technique itself. Lighting intensity and uniformity are typical examples. The user itself also influences the value of $d, \sigma_{u}, \sigma_{\varepsilon}$ and $\lambda$ since some settings impacting these values must be fixed. For DIC, the main influencing settings are the subset size and the order of the subset shape functions used to model the displacement within the subsets [9]. Another influencing parameter is the quality of the pattern itself. Some recent studies investigate this problem [10, 11, 12, 13, 14, 15] for instance. In particular, image gradient is the main parameter influencing sensor noise propagation to the final displacement maps [16, 17]. As a consequence, the authors of [13] claim that random patterns are not optimal in terms of sensor noise propagation, and that regular patterns such as checkerboards should theoretically be used instead, image gradient being the highest possible with this type of pattern if the latter is correctly sampled. Refs. [18] and [19] confirm that checkerboards lead to lower noise level in displacement maps than random speckles and classic 2D grids, respectively. Such a pattern is however periodic and not random, and the problem is that DIC may converge to wrong solutions in this case, the cost function to be minimized featuring many local minima. In [8], it is shown that minimizing the optical residual can advantageously be switched from the spatial to the Fourier domain in case of periodic patterns thanks to the Parseval's theorem and some additional calculations, see schematic view in Fig. 1.

Indeed, in addition to lower noise level, periodic patterns have some benefits compared to random patterns:

- the minimization of the optical residual is quasi-direct since a closed-form solution is available for the sought displacement field. The corresponding calculations can therefore be performed by hand in this case [8]. The solution found only depends on the phase distributions in the reference and the current images (see details below);

- the so-called pattern-induced bias which affects displacement and strain fields is lower (if not negligible) for periodic patterns than for random ones [20, 21, 18, 22]; 


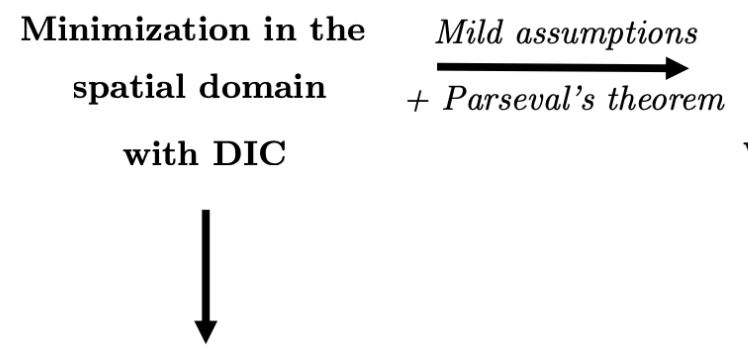

Minimization in the

Fourier domain

with a spectral method
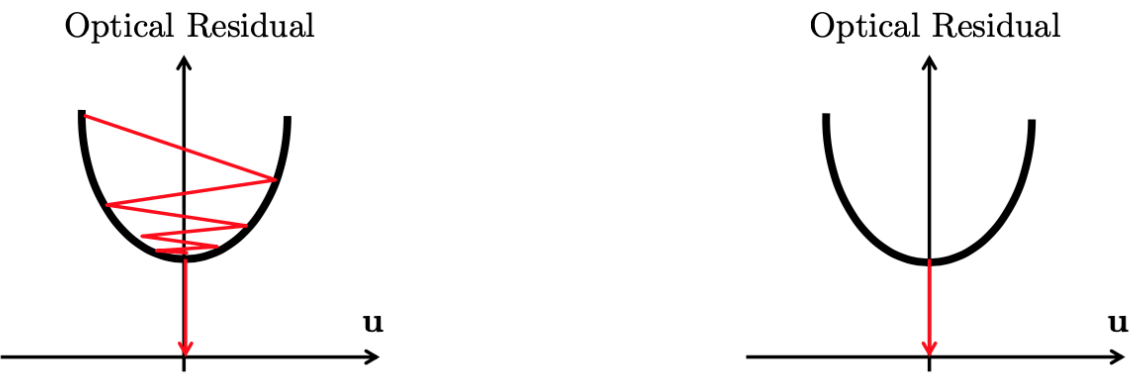

Figure 1: Schematic view of the minimization of the optical residual in the spatial domain with DIC, and in the Fourier domain with LSA, after the demonstration given in [8].

- whatever the domain which is considered to perform the minimization of the optical residual (the spatial domain or the Fourier domain), the information contained in the image of the current configuration must be mapped in the coordinate system of the reference configuration. This leads interpolation of the gray level distribution to be performed in the spatial domain, while a fixed-point algorithm written on the retrieved phase maps can be used in the Fourier domain. An important remark is that the gray level distribution concerned by interpolation in the spatial domain is generally much more fluctuating throughout a speckle image than the phase distribution concerned by the fixed-point algorithm in the Fourier domain. This leads interpolation error to occur mainly with DIC, as modeled with the well-known S-shaped bias, see [23] for instance. On the contrary, this error is negligible when the minimization of the optical residual is performed in the Fourier domain.

Several techniques are available to extract the phase distributions of the reference and current images (see the survey on spectral techniques suitable for periodic patterns in [24]). The so-called Localized Spectrum Analysis (LSA) is one of these techniques. It will be used in this study. Indeed, it is shown in [24] that it gives a good compromise between final metrological performance and calculation time.

To sum up, the main errors that occur when processing periodic patterns with LSA are the systematic error due to the attenuation of the signal for the highest spatial frequencies involved in the displacement and strain maps, and the random error due to sensor noise propagation. In this context, the aim of this study is to focus on a parameter, which still 
remains largely underexplored in the literature. This parameter is the sampling density of the pattern. Examining the influence of the sampling density is relevant in a context for which the size of camera sensors continuously increases, so users can legitimately wonder to what extent this parameter plays a role on the quality of the results. We examine here more specifically the influence of this parameter on the noise level in the final displacement maps. Only a limited number of papers tackle the influence of the sampling density of the pattern on the quality of the results. The author of [25] claims that 3 pixels per dot (in average) should be employed in the case of random speckles. [26] partially discusses this question for periodic patterns from a theoretical/numerical point of view, but no experimental result is given.

Strictly speaking, the sampling density is the number of pixels per unit length, which is used to sample a signal. For the sake of convenience, we consider here the number of pixels per period, the patterns under consideration being periodic. This quantity is denoted hereafter by $\rho$.

We focus here our attention on the checkerboard pattern since it is expected to be optimal in terms of sensor noise propagation. We also take this opportunity to examine the influence of the number of pixels per period $\rho$ on noise propagation with classic $2 \mathrm{D}$ grids, such patterns being still used even though they are not optimal. Closed-form expressions are available to estimate a priori the noise level in displacement and strain maps deduced from periodic patterns by using LSA. These formulas were established under some assumptions, in particular the fact that the signal (here the gray level distribution) is continuous [27], which is not the case in real images. These formulas were only partially experimentally verified in [28], in the sense that the effect of sampling was not investigated and that only classic 2D grids were studied.

In this context, the objective of this paper is to examine in depth the influence of $\rho$, on the noise level in displacement and strain maps retrieved from images of checkerboard and $2 \mathrm{D}$ grid patterns. We also study to what extent predictive formulas for noise propagation to displacement and strain maps are valid in these cases. The noise level obtained in both cases is also compared.

The paper is organized as follows. The fundamentals of LSA are first briefly recalled. The experimental setup is then presented. The effect of sampling is experimentally evidenced, discussed and modeled. The patterns considered here are a checkerboard and a classic 2D grid, both featuring the same period. Notations used in this paper are gathered in Table 1.

\section{A brief reminder on the Localized Spectrum Analysis}

\subsection{Localized Spectrum Analysis}

When a spectral technique is used to minimize the optical residual, the displacement field can be expressed as a function of the phase field of the reference image and its counterpart of the current one. Strain fields are then deduced by differentiation. This link between phase 


\begin{tabular}{|c|c|c|}
\hline Symbol & SI Unit (non-SI Unit) & Definition \\
\hline$\alpha$ & $(\mathrm{rad})$ & $\begin{array}{l}\text { Angle between the horizontal axis and the first direction } \\
\text { along which the WFT is calculated }\end{array}$ \\
\hline$\varepsilon_{i j}$ & {$[-]$} & $i j$ strain, $i j=x x, x y, y y$ \\
\hline$\lambda$ & [-] & Bias \\
\hline$\Phi^{\text {cur }_{i}}$ & $(\mathrm{rad})$ & Phase of the current image along direction $i$ \\
\hline$\Phi_{i}^{r e f}$ & $(\mathrm{rad})$ & Phase of the reference image along direction $i$ \\
\hline$\rho$ & (pixel) & Number of pixels per period $p^{\prime}$ \\
\hline$\sigma_{\varepsilon}$ & {$[-]$} & Standard deviation of the noise affecting the strain maps \\
\hline$\sigma_{\Phi}$ & $(\mathrm{rad})$ & Standard deviation of the noise affecting the phase maps \\
\hline$\sigma_{\Phi^{\prime}}$ & {$\left[\mathrm{m}^{-1}\right] \quad\left(\mathrm{rad} \mathrm{m}^{-1}\right)$} & $\begin{array}{l}\text { Standard deviation of the noise affecting the phase } \\
\text { derivative maps }\end{array}$ \\
\hline$\sigma_{\text {image }}$ & (gray level count) & Standard deviation of the noise affecting any image \\
\hline$\sigma_{u}$ & [m] & $\begin{array}{l}\text { Standard deviation of the noise affecting the } \\
\text { displacement maps }\end{array}$ \\
\hline$\theta$ & $(\mathrm{rad})$ & $\begin{array}{l}\text { Angle between the horizontal axis and any direction } \\
\text { along which the WFT is calculated }\end{array}$ \\
\hline$c$ & [m] & Side of the square zone covered by one pixel on the specimen \\
\hline$d$ & [m] & Spatial resolution of the technique for a bias $\lambda$ \\
\hline$D$ & {$[\mathrm{~m}]$} & Distance between specimen and camera \\
\hline$f$ & [m] & Fundamental frequency of the periodic pattern \\
\hline$K$ & (gray level count) & Modulus of $\widehat{s_{w}}$ \\
\hline$\ell$ & {$[\mathrm{m}]$} & Standard deviation of the Gaussian envelope used in the WFT \\
\hline$p$ & [m] & $\begin{array}{l}\text { Period of the pattern along the direction where the WFT } \\
\text { is calculated }\end{array}$ \\
\hline$p^{\prime}$ & {$[\mathrm{m}]$} & Period of the pattern along the $x^{\prime}$ and $y^{\prime}$ \\
\hline$s$ & (gray level count) & Signal or spatial gray level distribution \\
\hline$\widehat{s_{w}}$ & (gray level count) & Windowed Fourier Transform of $s$ \\
\hline$u_{i}$ & {$[\mathrm{~m}]$} & Displacement along direction $i, i=x, y$ \\
\hline$x_{p x}$ & $($ pixel $)$ & Any length $x$ expressed in pixel after dividing $x$ by $c$ \\
\hline$w$ & {$\left[\mathrm{~m}^{-2}\right]$} & Gaussian window used in the Windowed Fourier Transform \\
\hline
\end{tabular}

Table 1: Main notations used in the paper

and displacement writes as follows:

$$
u_{i}(x, y)=-\frac{p}{2 \pi}\left(\Phi_{i}^{c u r}\left(x+u_{x}(x, y), y+u_{y}(x, y)\right)-\Phi_{i}^{r e f}(x, y)\right) \quad i \in\{x, y\} .
$$

In this equation, $p$ is the period of the periodic pattern, $\Phi_{i}^{r e f}$ and $\Phi_{i}^{c u r}, i \in\{x, y\}$, are the phase distributions of this periodic pattern in the reference and current configurations, respectively. $u_{x}$ and $u_{y}$ are the displacements along $x$ and $y$, respectively. It turns out that the displacement $u_{i}, i \in\{x, y\}$, is determined if the phases of the reference and current images $\Phi_{i}^{r e f}$ and $\Phi_{i}^{c u r}, i \in\{x, y\}$, along $x$ and $y$ are known. It can be seen that the unknown components of the displacements are involved in both parts of Eq. 1. A fixed-point algorithm 
is classically used to find this displacement, which gives

$$
\left\{\begin{aligned}
u_{i}^{(0)}(x, y) & =-\frac{p}{2 \pi}\left(\Phi_{i}^{c u r}(x, y)-\Phi_{i}^{r e f}(x, y)\right) \quad i \in\{x, y\} \\
u_{i}^{(n+1)}(x, y) & =-\frac{p}{2 \pi}\left(\Phi_{i}^{c u r}\left(x+u_{x}^{(n)}(x, y), y+u_{y}^{(n)}(x, y)\right)\right. \\
& \left.-\Phi_{i}^{r e f}(x, y)\right) \quad i \in\{x, y\} .
\end{aligned}\right.
$$

The phases at non-integer coordinates are obtained by interpolation of the phases at integer coordinates. Convergence is generally obtained in one iteration, so the procedure is very fast. More detail on this fixed-point algorithm and on the procedure used to process displacement greater than the period of the pattern can be found in [29].

LSA is one of the techniques available to obtain $\Phi_{i}^{c u r}$ and $\Phi_{i}^{r e f}, i \in\{x, y\}$. With this technique, the first step is to calculate the windowed Fourier transform (WFT) of the signal (here the gray level distribution) $s$. This quantity is denoted by $\widehat{s_{w}}(x, y, \theta)$. The WFT is calculated for only one spatial frequency $f$ which is equal to the nominal frequency $f=\frac{1}{p}$ of the periodic pattern, and for a direction defined by an angle $\theta$ with respect to the horizontal axis as explained below. Thus

$$
\widehat{s_{w}}(x, y, \theta)=\int_{-\infty}^{+\infty} \int_{-\infty}^{+\infty} s(\eta, \xi) w(x-\eta, y-\xi) e^{-2 i \pi f(\eta \cos \theta+\xi \sin \theta)} d \eta d \xi
$$

In this equation, $w$ is a window centered at the pixel of coordinates $x, y$ where $\widehat{s_{w}}(x, y, \theta)$ is calculated. The Gaussian window constitutes the best tradeoff between various constraints [26], so it is chosen here. The function defining this Gaussian window is given by the following equation:

$$
w(x, y)=\frac{1}{2 \pi \ell^{2}} e^{\left(-\frac{x^{2}+y^{2}}{2 \ell^{2}}\right)}
$$

where $\ell$ is the standard deviation of the Gaussian function. Similarly to the subset size in DIC, $\ell$ can be considered as a handy parameter which governs the apparent width of the window used in LSA. The Gaussian function has no compact support, so the apparent width is arbitrarily equal to $6 \ell$ according to the so-called " $3-\sigma$ rule" [30]. In Eq. $3, \theta$ represents the angle of the direction along which the WFT is calculated. To obtain bidimensional displacement, this WFT shall be calculated along two directions, namely $\theta=\alpha+\frac{\pi}{4}$ and $\theta=\alpha+\frac{3 \pi}{4}$ for a checkerboard [19], and $\theta=\alpha$ and $\theta=\alpha+\frac{\pi}{2}$ for a classic 2D grid [29]. $\alpha$ is the angle which gives the orientation of the natural axes of symmetry of the periodic pattern with respect to the horizontal border of the images, see the definition of these axes in Fig. 2. $\alpha \neq 0$ here in order to avoid aliasing in the images, [31]. A mere change of basis finally gives the phases in the $(x, y)$ coordinate system. To sum up, the same image processing is applied for $2 \mathrm{D}$ grid and checkerboard but: 


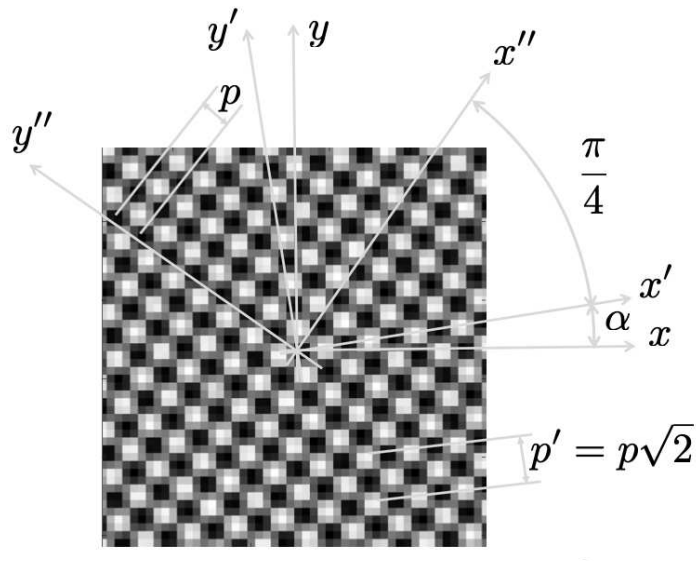

(a) Checkerboard, $\theta=\alpha+\frac{\pi}{4}, \theta=\alpha+\frac{3 \pi}{4}$ and $p=p^{\prime} \frac{\sqrt{2}}{2}$

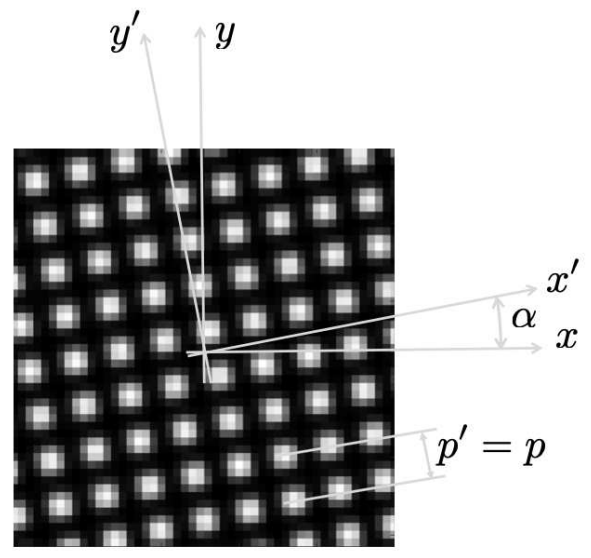

(b) $2 \mathrm{D}$ grid, $\theta=\alpha, \theta=\alpha+\frac{\pi}{2}$ and $p=p^{\prime}$

Figure 2: Closeup view of the two patterns under study. Period $p^{\prime}$ is the same in both cases.

- with a $2 \mathrm{D}$ grid, the directions of the lines along $x^{\prime}$ and $y^{\prime}$ are directly considered to define $\theta$ instead of the lines and columns of diamonds that can be observed along $x^{\prime \prime}$ and $y^{\prime \prime}$ for a checkerboard;

- the consequence is that the period $p$ used in the WFT is $\sqrt{2}$ times lower for a checkerboard than for a $2 \mathrm{D}$ grid, even though the period $p^{\prime}$ is the same for both types of pattern (see the difference between $p$ and $p^{\prime}$ in Fig. 2).

As explained in [24], LSA is a particular case of the so-called Windowed Geometric Phase Analysis (WGPA) [32, 33], in the sense that only one frequency is considered, whereas the whole "dot" surrounding the nominal frequency in the Fourier domain is employed with WGPA. The benefit is that the sought phases, which eventually give the displacement according to Eq. 1, are considered to be directly the arguments of the WFT calculated for $\theta=0$ and $\theta=\frac{\pi}{2}$ plus a constant value denoted here by $\kappa[27]$ (this constant value vanishes when subtracting current and reference phases), while these phases are determined after performing an additional inverse Fourier transform with WGPA. This simplified approach leads the highest spatial frequencies involved in the displacement or strain maps to be attenuated because each actual phase is not exactly equal to the argument of the corresponding WFT plus $\kappa$, but to this argument convolved by the window $w$ used in the WFT [27] plus $\kappa$. This latter effect can be partially counterbalanced by using a suitable deconvolution procedure described in [34]. An advantage of LSA is that the computing time to get the displacement field from a pair a reference and current images is much lower than with WGPA [24]. Finally, closed-form expressions for the systematic error and for the random error due to sensor noise 
propagation are available for LSA. This latter point is recalled specifically in the following section.

\subsection{Sensor noise propagation}

Random errors which affect displacement and strain maps are mainly due to sensor noise, which propagates when phases are extracted from noisy images. This propagation has been studied in [27] and predictive formulas giving the standard deviation of the noise in the phase and phase derivative maps have been given. These quantities, denoted here by $\sigma_{\phi}$ and $\sigma_{\phi^{\prime}}$, respectively, also represent the phase and phase derivative resolutions according to the definition given in Appendix A. These predictive formulas write as follows [27]:

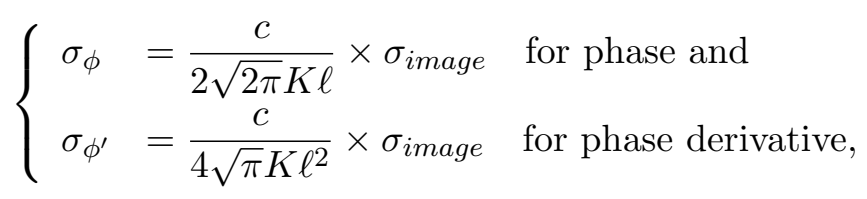

where $K$ is the modulus of the WFT defined in Eq. 3 above, $c$ the size of the square covered by a pixel on the surface under investigation, and $\sigma_{\text {image }}$ the standard deviation of the noise affecting the image. This noise is assumed here to be homoscedastic. This is quite a rough assumption since noise in real images is heteroscedastic [35] (it is indeed signal-dependent). $K$ is defined by [27]:

$$
K \simeq \frac{\left|d_{1}\right| \gamma A}{2}
$$

where $d_{1}$ is the first non-zero coefficient in the Fourier expansion of the profile of the periodic signal. In other words, this quantity is linked to the "closeness" of this function to a pure sine profile, as demonstrated in [27]. $A$ is the amplitude of the periodic signal, and $\gamma$ the contrast of the signal. This latter quantity generally gently changes throughout the images because of the unavoidable non-uniformity of the lighting. This does not affect, however, the validity of our approach which is based on window analysis. The contrast can indeed reasonably be assumed constant within the analysis window. The propagation of the noise in displacement and strain maps is then deduced by using Eq. 2, further iterations in the fixed-point algorithm used to deduce $u_{x}$ and $u_{y}$ from the initial phases in Eq. 1 being assumed not to significantly affect the results.

Bearing in mind that two phase (resp. phase derivative) maps are needed to get one displacement (resp. strain) map and that these two phase maps are noisy, one can deduce from Eq. 2 and 5 the standard deviation of the noise affecting displacement and strain maps. By definition (see Appendix A), these quantities are equal to the displacement resolution $\sigma_{u_{i}}$, $i=x, y$ and to the strain resolution $\sigma_{\varepsilon_{i j}}, i, j=x, y$ :

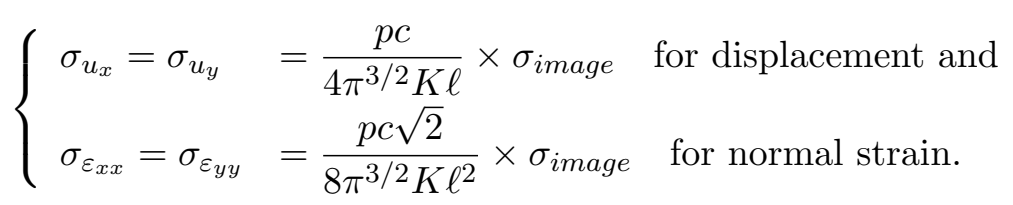


According to the definition of $\varepsilon_{i j}, i, j=x, y\left(\varepsilon_{x y}=\frac{1}{2}\left(\frac{\partial u_{i}}{\partial x_{j}}+\frac{\partial u_{j}}{\partial x_{i}}\right)\right)$, it can easily be checked that $\sigma_{\varepsilon_{x y}}$ is $\sqrt{2}$ times lower than $\sigma_{\varepsilon_{x x}}$ and $\sigma_{\varepsilon_{y y}} \cdot \sigma_{u_{x}}$ and $\sigma_{u_{y}}$ have the same unit as the displacement (meter). However, these quantities will be given in $\mathrm{mm}$ in 4 below for an easier comparison with the period of the periodic patterns studied here, which is a fraction of a millimeter. Finally, since we focus here on the influence of $\rho$ on the results, this quantity is introduced by writing that the size $c$ of the square covered by one pixel on the specimen is equal to $c=\frac{p^{\prime}}{\rho}$. Thus

$$
\left\{\begin{array}{cl}
\sigma_{u_{x}}=\sigma_{u_{y}}=\frac{p p^{\prime}}{4 \pi^{3 / 2} K \ell \rho} \times \sigma_{\text {image }} & \text { for displacement and } \\
\sigma_{\varepsilon_{x x}}=\sigma_{\varepsilon_{y y}}=\frac{p p^{\prime} \sqrt{2}}{8 \pi^{3 / 2} K \ell^{2} \rho} \times \sigma_{\text {image }} & \text { for normal strain, }
\end{array}\right.
$$

with $p^{\prime}=p \sqrt{2}$ for checkerboard, and $p^{\prime}=p$ for grid, see Fig. 2. Again and for the same reason as above, $\sigma_{\varepsilon_{x y}}$ is $\sqrt{2}$ times lower than $\sigma_{\varepsilon_{x x}}$ and $\sigma_{\varepsilon_{y y}}$.

Eq. 8 predict that noise is inversely proportional to $\rho$. At first sight, this is intuitively logical. Indeed, noise is progressively averaged out as $\rho$, and thus the number of pixels covered by the Gaussian window $w$ used in the WFT, increases. If $w$ covers a square zone containing $n \times n$ pixels, each of them being affected by a homoscedastic noise of standard deviation denoted by $\sigma_{\text {image }}$, calculating the weighted average of the signal over this zone gives a result affected by a noise of standard deviation $\sigma_{\text {image }}^{\text {filtered }}=\frac{\sigma_{\text {image }}}{n} \times S$, with $S=$ $\sqrt{\sum_{i=1}^{n} \sum_{j=1}^{n} w^{2}\left(x_{i}, y_{j}\right)} \cdot S^{2}$ is here nothing but the discrete form of integral $I$ defined by

$$
I=\int_{-\infty}^{+\infty} \int_{-\infty}^{+\infty} w^{2} d x d y
$$

Thus

$$
\begin{aligned}
I & =\left(\frac{1}{2 \pi \ell^{2}}\right)^{2} \int_{-\infty}^{+\infty} \int_{-\infty}^{+\infty} e^{\left(-\frac{x^{2}+y^{2}}{\ell^{2}}\right)} d x d y \\
& =\frac{1}{4 \pi \ell^{2}},
\end{aligned}
$$

and

$$
\begin{aligned}
\sigma_{\text {image }}^{\text {filtered }} & =\frac{\sigma_{\text {image }}}{n} \times \sqrt{\sum_{i=1}^{n} \sum_{j=1}^{n} w^{2}\left(x_{i}, y_{j}\right)} \\
& \simeq \frac{\sigma_{\text {image }}}{n} \times \frac{1}{2 \sqrt{\pi} \ell} .
\end{aligned}
$$


So $\sigma_{u_{i}}, i=x, y$ and $\sigma_{\varepsilon_{i j}}, i, j=x, y$ on the one hand, and $\sigma_{\text {image }}^{\text {filtered }}$ on the other hand, are all inversely proportional to $\rho$ since $n$ is proportional to $\rho$ when the WFT is calculated. The object of the following sections is to check if this theoretical prediction is experimentally verified.

\section{$3 \quad$ Experimental setup and image processing}

\subsection{Experimental setup}

The experiments performed in this study were carried out with a SENSICAM QE CCD camera featuring a 12-bit/1040 × 1376-pixel sensor. A Peltier cooler is embedded in this camera, near the sensor. The advantage of this cooler is to stabilize the temperature during the whole experiment, and thus sensor noise amplitude since temperature and noise level are closely related. A Tokina Macro lens with a focal length equal to $100 \mathrm{~mm}$ was mounted on the camera. It is worth noting that this lens is dedicated to large sensors of size $24 \times 36 \mathrm{~mm}^{2}$. Since the sensor of the camera used in this study is smaller in size, it means that the central part of the lens, thus its best part in terms of optical distortion and vignetting, is used here. The experimental setup is shown in Fig. 3. The camera was fixed onto a metallic frame. Only one LED light source placed on the right-hand side of the setup was employed, but a mirror placed on the left-hand side ensured a nearly uniform lighting of the pattern. No flickering occurred while taking the images, thus avoiding additional processing to properly estimate noise in the images [36].

Two specimens marked each with a different pattern were considered. The first pattern was a checkerboard and the second a classic 2D grid. Closeup views of both patterns are showed in Fig. 2. These patterns were deposited onto the specimens by using the procedure described in [37]. The period $p^{\prime}$ of both patterns was equal to $p^{\prime}=0.2 \mathrm{~mm}$. It means that both the size of the squares forming the checkerboard and the width of the lines of the 2D grid were equal to $0.1 \mathrm{~mm}$. These specimens were fixed onto a support allowed to move along the horizontal $y$-direction thanks to a slide linkage. The movement was controlled by a screw, see Fig. 3.

25 values of the distance $D$ between camera and specimen were considered, each distance leading to a different value of $\rho$. For each value of $D, \rho$ was estimated by using the following procedure:

1. taking the Fourier transform of the grid or checkerboard image in the reference configuration

2. determining the coordinates of the highest spike of the spectrogram (excluding the origin)

3. deducing the mean frequency of the periodic pattern by calculating the distance in pixel $^{-1}$ between the origin and this spike after scaling the axes to account for possible differences in dimensions along the two directions of the pattern image 


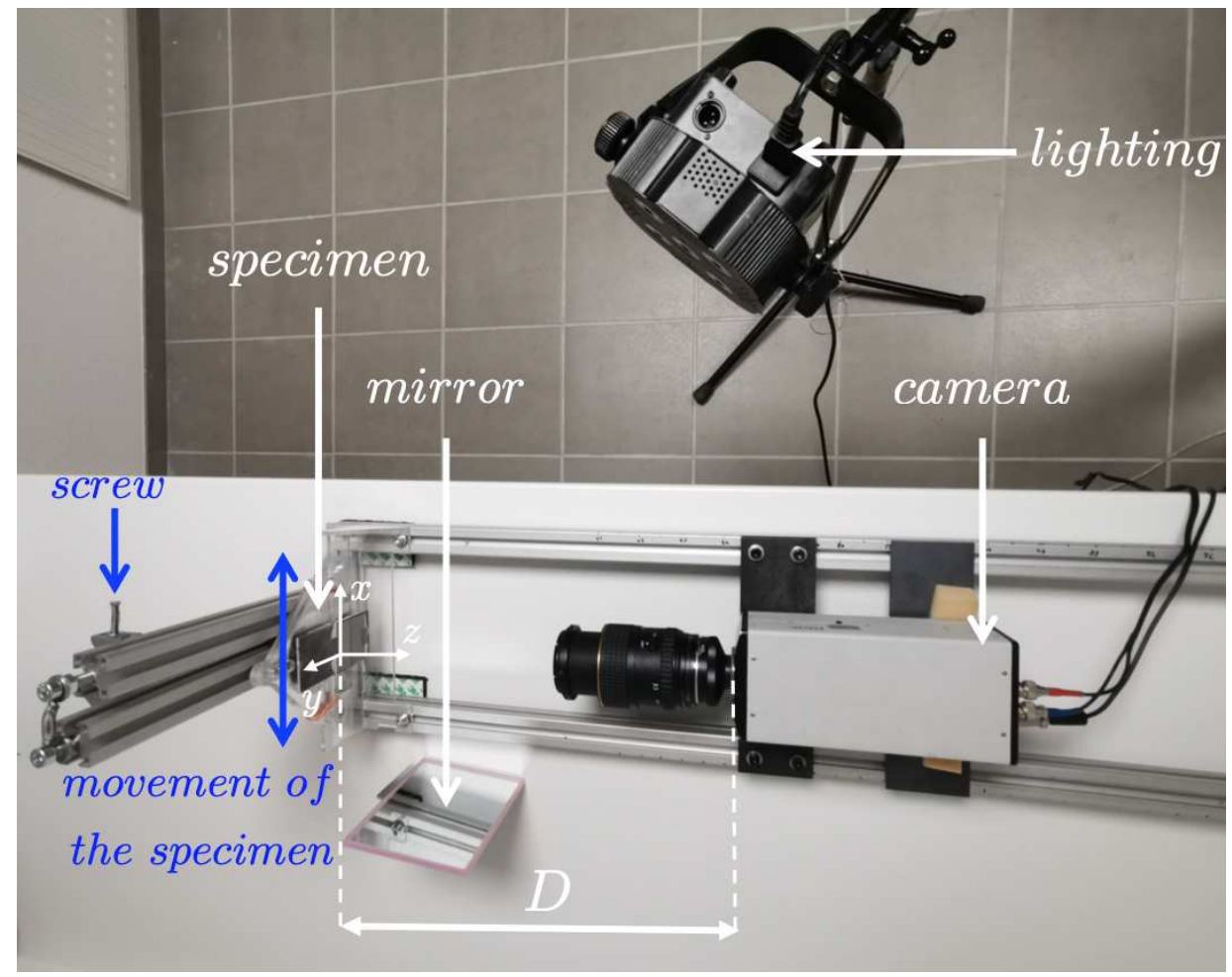

Figure 3: Top view of the experimental setup.

4. deducing the period in pixel by taking the inverse of this frequency.

For each value of $D, 200$ pairs of images (reference and after translation) were shot and processed with LSA. This gave 200 displacement fields and 200 strain fields after differentiation. This differentiation was performed by using the "gradient function" of Matlab, which implements a central difference scheme relying on a spacing between points equal to two pixels. Special attention was paid to being sure that fluctuations in those fields were only due to sensor noise propagation. The procedure developed to reach this goal is described in the following section.

\subsection{Removing the effects of vibrations}

\subsubsection{Principle}

We assume that the displacement maps are estimated from two images of a non-deformed plane specimen. Therefore, the observed displacement $\left(u_{x}(x, y), u_{y}(x, y)\right)$ comes from sensor noise, which propagates through the estimation process, but also from a rigid body movement (RBM) which is due to

1. the translation along the $y$-direction applied through the screw; 

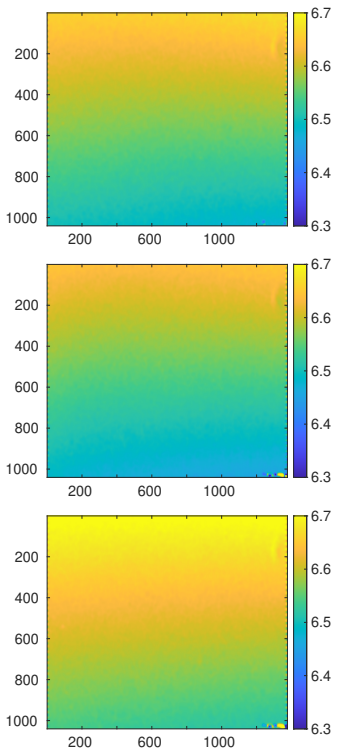

1- $x$-displacement raw result
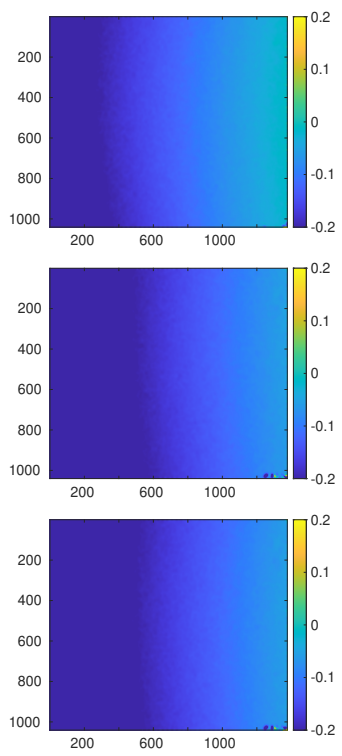

2- $y$-displacement raw result
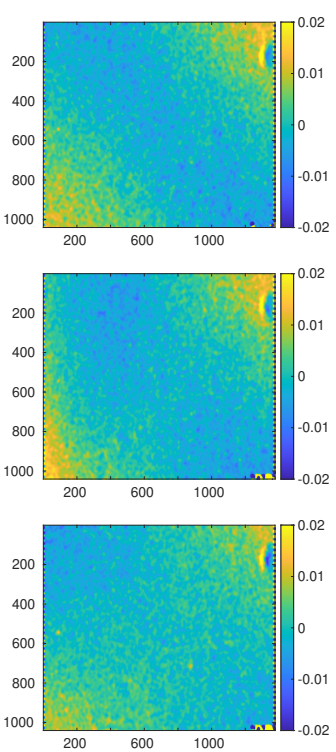

3- $x$-displacement after RBM removal
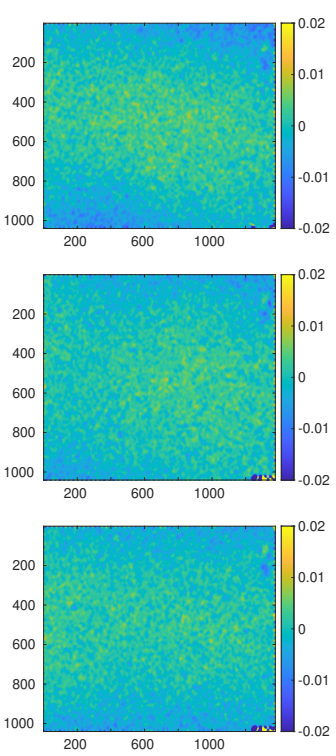

4- $y$-displacement after RBM removal

Figure 4: Each row shows for a given pair of images the $x$ - and $y$-displacement field (in pixels) before and after removing the effect of RBM. Here, the two pairs come from a series of images where the camera is at 50 centimeters of the specimen. The shutter time is 24 milliseconds and the specimen is supposed to be immobile.

2. a slight rotation because joint clearance causes the linkage between specimen and metallic frame not to be rigorously a slide linkage;

3. the effect of vibrations. Indeed, it has been observed that the Peltier cooler induced slight vibrations. Even though the camera is firmly attached to the metallic frame, these vibrations cause a slight movement of the camera to occur with respect to the observed specimen.

Since the specimen is plane, it is known that, in the pinhole camera model, RBM gives a homography transform $H$ between the two images from which the displacement map is estimated [38]. However, in our experimental setting, the focal length of the camera is large compared to the size of the imaged specimen. Moreover, the out-of-plane rotation of the specimen is very small, since it is only caused by vibrations or by the presence of clearance in the slide linkage. We can thus safely simplify the pinhole camera model into the affine camera model, for which two images of a static specimen are related by an affine transformation. This is a common assumption in computer vision, see [39] for instance.

In order to compute statistics on the part of the displacement related to noise, we have to cancel out the affine transformation related to RBM.

Let us denote $(x, y)$ the pixel coordinate of a solid point in the first image, and $\left(x^{\prime}, y^{\prime}\right)$ 
the corresponding pixel coordinate of the same point in the second image. The following equation holds from the definitions and hypothesis above. On the one hand,

$$
\left\{\begin{array}{l}
x^{\prime}=x+u_{x}(x, y) \\
y^{\prime}=y+u_{y}(x, y)
\end{array}\right.
$$

and, on the other hand,

$$
\left\{\begin{array}{l}
x^{\prime}=x+a_{11} x+a_{12} y+a_{13}+n_{x}(x, y) \\
y^{\prime}=y+a_{21} x+a_{22} y+a_{23}+n_{y}(x, y)
\end{array}\right.
$$

under the affine transformation assumption. Here, $n_{x}$ and $n_{y}$ are random variables modeling the random part of the estimated displacement caused by sensor noise propagation.

The parameters of the affine transformation can be estimated in the least square sense by minimizing the two functions $\sum_{x, y}\left|a_{11} x+a_{12} y+a_{13}-u_{x}(x, y)\right|^{2}$ and $\sum_{x, y} \mid a_{21} x+a_{22} y+a_{23}-$ $\left.u_{y}(x, y)\right|^{2}$ with respect to $\left(a_{11}, a_{12}, a_{13}\right)$ and $\left(a_{21}, a_{22}, a_{23}\right)$, respectively. In spite that $n_{x}$ and $n_{y}$ are correlated noises, we still use the classic optimal least square estimator instead of the generalized least square estimator which would require to estimate the covariance matrices of $n_{x}$ and $n_{y}$ [40].

Now, $n_{x}^{\prime}$ and $n_{y}^{\prime}$ are defined for any $(x, y)$ as:

$$
\left\{\begin{array}{l}
n_{x}^{\prime}(x, y)=u_{x}(x, y)-\left(a_{11} x+a_{12} y+a_{13}\right) \\
n_{y}^{\prime}(x, y)=u_{y}(x, y)-\left(a_{21} x+a_{22} y+a_{23}\right) .
\end{array}\right.
$$

These quantities are estimators of the random parts $n_{x}$ and $n_{y}$ in the $x$ and $y$ components of the displacement maps, respectively. The standard deviation of these random quantities was determined at any pixel, and then served to determine the empirical displacement resolutions $\sigma_{u_{x}}$ and $\sigma_{u_{y}}$ along $x$ and $y$, respectively. Let us now examine the effect of this correction on the apparent noise in displacement maps.

\subsubsection{Illustration of the effect of RBM removal}

Fig. 4 illustrates the effect of RBM (among which vibrations) as well as the impact of the procedure proposed above to remove it from the displacement maps. The raw estimations of the $x$ - and $y$-displacement fields are shown in columns 1 and 2 of this figure. These displacements have large values with a gently varying distribution caused by RBM. The $u_{x}$ displacement is nearly an affine function of $y$, and the $u_{y}$ displacement an affine function of $x$, which means that the specimen has been subjected to a translation and a rotation. This is presumably due to the fact that because of clearance in the sliding linkage, the specimen slightly rotated while a translation was imposed along the $x$-axis. As a result, the $x$ displacement is not constant and not equal to the imposed translation, and the $y$-displacement is not strictly equal to 0 . In addition to this slight rotation, vibrations add some small random perturbations. This explains why the displacement fields exhibit slight fluctuations along the 
series. As explained before, the effect of RBM was removed by subtracting the affine part from all the displacement maps retrieved from the images of the series. It can be seen in column 3 and 4 of Fig. 4 that the displacement obtained after correction is an almost uniformly distributed random displacement field, although a slight residual effect can still be observed.
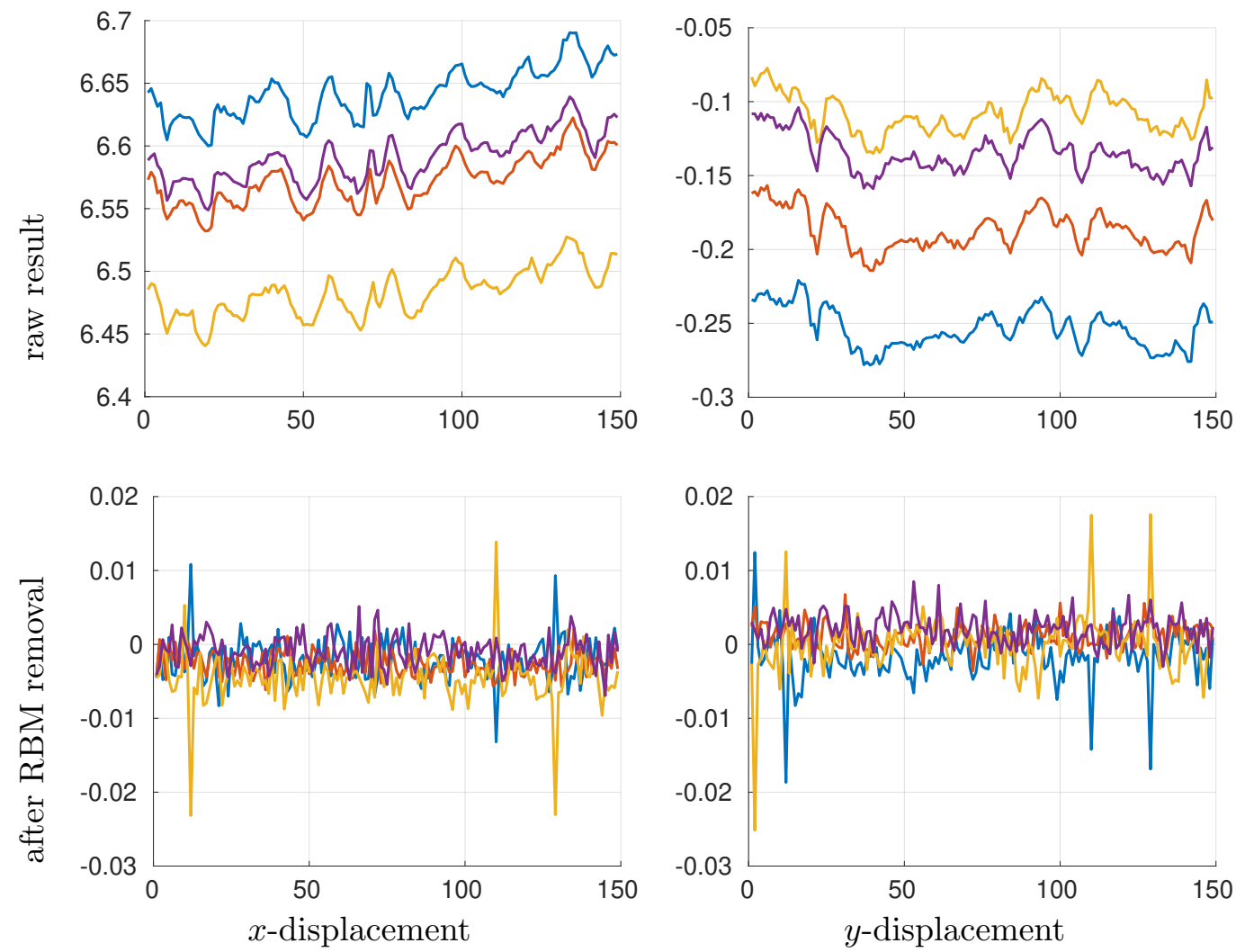

Figure 5: Evolution of the displacement (in pixels) measured at four distant point of the specimen surface along time. Note the spatial correlation of the raw measurement, which is corrected in a satisfactory manner after RBM removal.

Fig. 5 is another illustration of the effect of RBM. The first row shows the evolution of the displacement measured at four distant points on the surface of the specimen. In-phase fluctuations can be observed. They are due to the random vibrations which affect image acquisition. After the proposed RBM removal procedure, the measurements shown in the second row are no longer temporally correlated, as can be expected if sensor noise is the main phenomenon causing these fluctuations.

Finally, Fig. 6 shows the distribution of the temporal standard deviation of the displacement over the surface of the specimen. The standard deviation of the raw result is larger along the $x$-direction than along the $y$-direction. The reason is probably that because of the sliding linkage, the amplitude of the vibrations is larger along $x$ than along $y$. The standard deviation is much smaller after removing the effect of RBM. It also has the same amplitude 

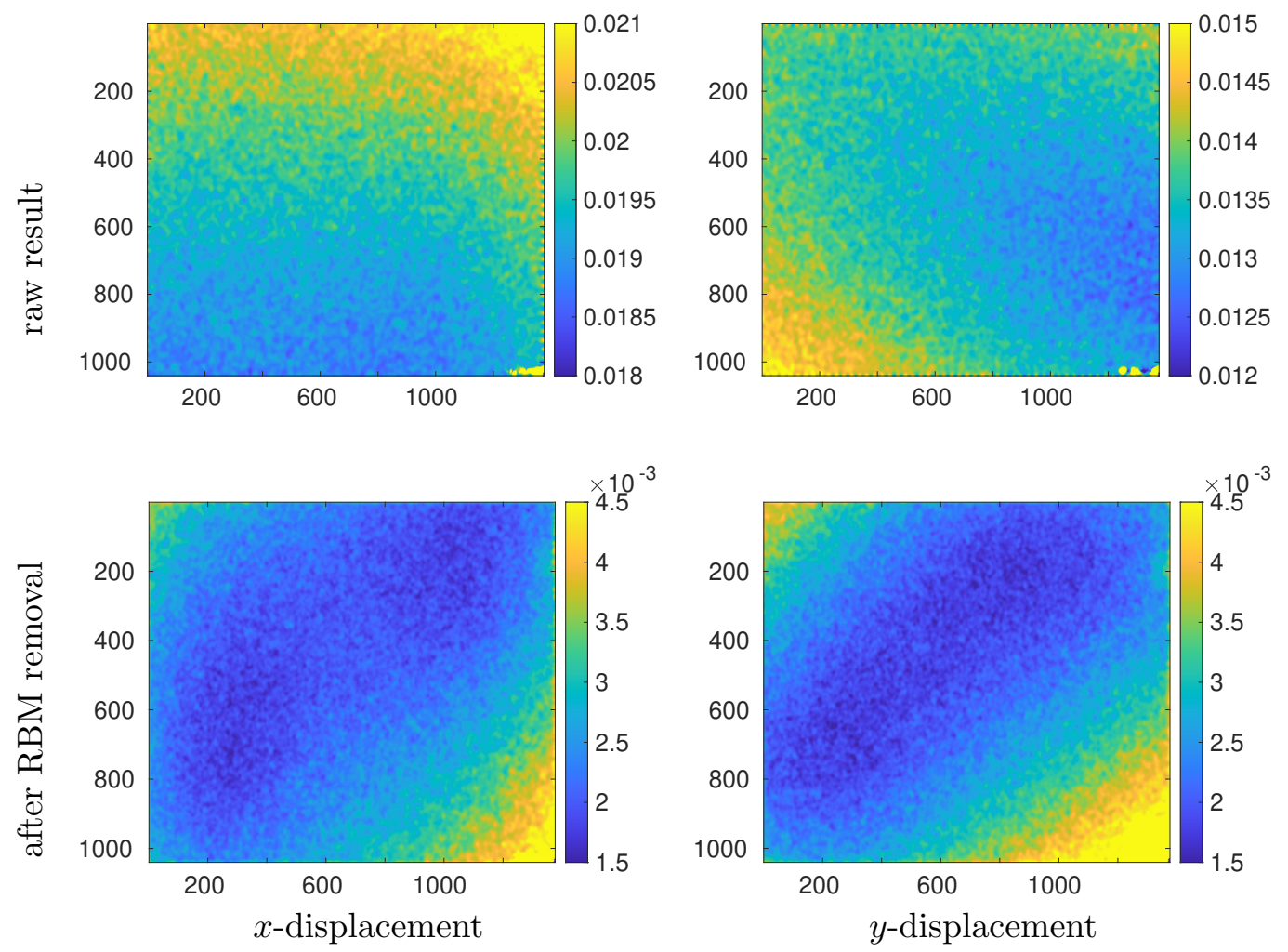

Figure 6: Distribution of the temporal standard deviation of the displacement (in pixels) over the specimen surface, calculated without and with RBM removal.

along both directions. It is not rigorously uniform. This is certainly due to the non-uniformity of the light, which then causes sensor noise to spatially change in amplitude, this noise being heteroscedastic [35].

\subsection{Estimating the noise parameters of the camera}

Since sensor noise propagation is studied here, the noise in the images had also to be properly estimated. As explained in [35] for instance, noise affecting images is heteroscedastic, with a model such that the variance is an affine function of the brightness, thus

$$
\sigma_{\text {image }}^{2}(x, y)=a s(x, y)+b
$$

where $a$ and $b$ are two constants which depend on the camera sensor itself. These quantities are provided by the camera supplier [41]. They were also thoroughly identified in [42] for the camera used in this study. A sanity check was however performed here by using the following procedure. After deliberately defocusing the lens, different series of 200 images of a white background were taken with an aperture $f / 11$ and a shutter time $t \in$ $\{1,2,3,4,5,6,8,10,12,14,16,18,20,22,24\} \mathrm{ms}$, in such a way that the widest possible range 


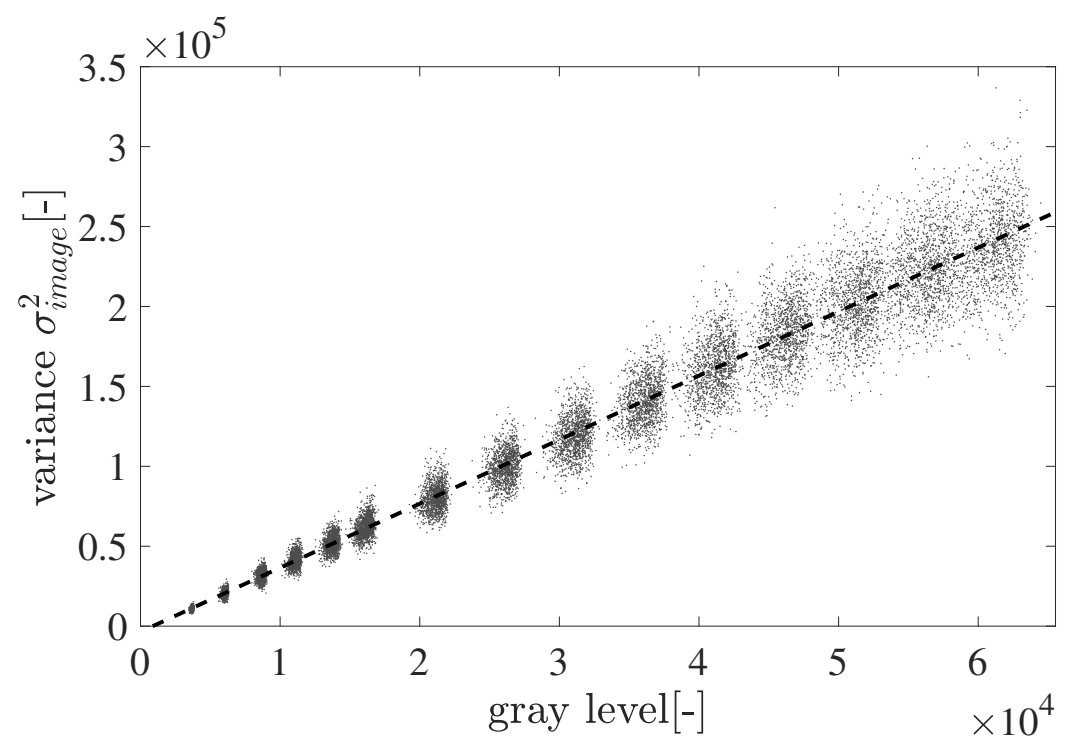

Figure 7: Variance of the noise at each pixel as a function of the gray level. Some fluctuations can be seen for each value of the shutter time because the noise properties are not rigorously uniform over the sensor field.

of gray levels was covered by the different sets of images, the illumination level being hold constant. The images were blurred in all cases to avoid having images corrupted by the vibrations mentioned above. Fig. 7 shows the point clouds found for each value of the shutter time. As expected, these point clouds are properly aligned in the gray level-variance plane. Their range also increases as the brightness increases. Such data enabled us to plot the line modeling the affine relationship between variance and gray level given in Eq. 17. Minimizing in the least squares sense the distance between model and experimental data, gives the value of $a$ and $b$, which are respectively the slope and the intercept of the line. The following values were found here: $a=4.01$ and $b=-3536$. The influence of $b$ on $\sigma_{\text {image }}(x, y)$ is negligible. It is worth noting that the value of $a$ found here is about half the value reported in [42], which is logical since the "gain low" option was used here while the "gain high" one was used in [42], a factor of two theoretically affecting the gain when switching from "high" to "low".

\subsection{Determining the empirical values of $\sigma_{i m a g e}, \sigma_{u_{i}}$ and $\sigma_{\varepsilon_{i j}}$}

Eq. 8 are formulas which predict the displacement and strain resolutions. They were established in $[27,28]$ and experimentally verified in [28] but only partially, since only a grid (and not a checkerboard) was used, and only a given value of $\rho$ was considered. The question here is to see if these equations are also verified for a checkerboard and for various values of $\rho$. Two problems arose however when assessing $\sigma_{i m a g e}, \sigma_{u_{i}}$ and $\sigma_{\varepsilon_{i j}}$ after processing the images: 
- micro-vibrations occurred during the experiments, which corrupted the characterization of the sensor noise affecting the images;

- $\sigma_{\text {image }}$ in Eq. 11 is not a constant throughout the images since actual noise is heteroscedastic, while Eq.11 were obtained assuming the noise was homoscedastic.

The first problem also occurred in [28]. It was resolved by eliminating the effect of vibrations by applying a so-called Non-Random Signal Removal (NRSR) procedure. This procedure is however only applicable when $\alpha$, which characterizes the rotation angle of the periodic pattern (see Fig. 2), is null. This is however not the case in the present study to avoid aliasing in the images [28]. The second problem was overcome by using a normalization of the noise obtained after removing the effect of vibrations. This normalization was performed by applying the so-called generalized Anscombe transform. The resulting noise becomes approximately homoscedastic after applying this transform defined in [43] after [44]. Since NRSR cannot be applied here, a simplified approach was used. This simplified approach is briefly explained below. First, we removed the effect of vibrations in the retrieved displacement maps by using the procedure described in 3.2. Second, we predicted the noise level in the images by using the model characterized in 3.3. Third, we consider a global value for $\sigma_{i m a g e}, \sigma_{u_{i}}$ and $\sigma_{\varepsilon_{i j}}$ instead of pixelwise distributions. It can be noted that, once sensor noise parameters are known, it is possible to apply the generalized Anscombe transform (GAT) to the acquired images so that the resulting noise is approximately homoscedastic. This would make it possible to use the predictive formula for $\sigma_{u_{i}}$ and $\sigma_{\varepsilon_{i j}}$ with a value of $\sigma_{\text {image }}$ constant and normalized over the image domain. In the present study, the two approaches (with and without GAT) were found to show only minimal differences. This was not the case in [42] because pixelwise distributions were considered instead of global values. For the sake of simplicity, we applied here the approach based on the global value of $\sigma_{\text {image }}$, and thus did not applied GAT.

Determination of $\sigma_{\text {image }}$ Coefficients $a$ and $b$ of the noise model defined in Eq. 17 being characterized by using the method described in 3.2 , it is possible, by using this model, to get a reasonable estimate of the sensor noise affecting a given image by applying Eq. 17. This standard deviation of the noise changing from one pixel to another since it depends on the brightness, a global estimation over any image of dimensions $M \times N$ can be obtained by calculating the following quantity

$$
\sigma_{\text {image }}=\sqrt{\frac{1}{N \times M} \sum_{k}^{N} \sum_{l}^{M}\left(\sigma_{\text {image }}\left(x_{k}, y_{l}\right)\right)^{2}}
$$

Determination of $\sigma_{u_{i}}$ and $\sigma_{\varepsilon_{i j}}$ In the same way, a global empirical estimation of the displacement and strain resolutions over a map of dimensions $M \times N$ can be defined by the 
following quantities:

$$
\left\{\begin{array}{l}
\sigma_{u_{i}}=\sqrt{\frac{1}{N \times M} \sum_{k}^{N} \sum_{l}^{M}\left(\sigma_{u_{i}}\left(x_{k}, y_{l}\right)\right)^{2}} \quad i=x, y \\
\sigma_{\varepsilon_{i j}}=\sqrt{\frac{1}{N \times M} \sum_{k}^{N} \sum_{l}^{M}\left(\sigma_{\varepsilon_{i j}}\left(x_{k}, y_{l}\right)\right)^{2}} \quad i j=x x, y y, x y
\end{array}\right.
$$

These empirical values for $\sigma_{i m a g e}, \sigma_{u_{i}}$ and $\sigma_{\varepsilon_{i j}}$ were used here to check the validity of the predictive Eq. 8 from a global point of view.

\section{Results}

\subsection{Preliminary tests: setting the aperture and shutter time of the camera}

Before taking images, the first point was to choose the aperture and shutter time of the camera. This combination of two parameters can be adjusted in such a way that the same amount of light hits any pixel of the sensor. This phenomenon is modeled by the so-called reciprocity law, which claims that if the aperture number (which is proportional to the inverse of the radius of the aperture of the diaphragm) is multiplied by a constant $k$, the shutter time should be multiplied by $k^{2}$.

However, the reciprocity law has practical limitations: the focal length is only approximately known (it may depend on the focus point for instance), and the aperture number and the shutter speed only take discrete pre-determined values. This is not a problem for generalpurpose photography but this prevents us from using this law to set the shutter speed as a function of the aperture. For a given aperture, the location of the right-hand tail of the gray level histogram was adjusted by fine-tuning the shutter time, which gave the experimental link between shutter time and aperture to keep a constant illumination. However, the point is that even though the same amount of light hitting the pixels can be obtained with several combinations of aperture and shutter time, changing the aperture influences the contrast in the images, see [45] for instance. It is shown in [45] that values which are optimal to get the highest contrast in the images (and thus the lowest noise in the displacement and strain maps according to Eqs. 8 and 6) are obtained for low values of the aperture. Fig. 8 illustrates this impact of the aperture by showing the amplitude of the gray level histogram as a function of $\rho$ for $f / 4, f / 5.6$ and $f / 8$. In each case, the shutter time was adjusted in such a way that the right-hand tail of the gray level histogram of the images was as close as possible to its maximum value, namely $2^{16}-1$ with tif images (the gray depth of the camera sensor is 12 bits, but tif images are always encoded with 8 or 16 bits). The location of the left-hand tail is then only governed by the sharpness of the images. The contrast reported in Fig. 8 is the difference between its maximum value, which was the same for all images $\left(2^{16}-1\right)$, and the minimum value defined by the location of the left-hand tail, which was picked by hand on the histograms. It is clear that the highest contrast is globally obtained with $f / 5.6$, which is consistent with the results given in [45]. Consequently, this aperture was chosen for all the results presented in the remainder of the paper. 
Two additional remarks can be drawn from this figure:

- the contrast in the images is an increasing function of $\rho$, but the contrast in the grid images is less influenced by $\rho$ than the contrast in the checkerboard images;

- the contrast is always lower for the checkerboard images, but the difference with the contrast in the grid images decreases as $\rho$ increases.

These phenomena are presumably due to the effect of the Point Spread Function (PSF) of the lens. Indeed, the gray level of the white pixels being always close to the maximum value, the gray level of the dark pixels (or the intensity of the black in the images, and thus the contrast) is found at the center of the dark squares forming the checkerboard. It is clear that the main impact of the PSF is to change the value of the gray level in the dark squares. For a given PSF, this phenomenon is all the more marked as $\rho$ is small. In addition, this phenomenon is more marked with a checkerboard since in this case, a dark square is bordered by white pixels along its four sides while for a grid, dark squares at the crossing between black lines are surrounded by black pixels along their four borders, white pixels bordering these dark squares only at their four corners. The conclusion is that the PSF plays a lower role for the grid than for the checkerboard, which explains the highest sensitivity to $\rho$ of checkerboard observed in Fig. 8. This assumption concerning the role of the PSF should be investigated further by using a lens with a different resolution power than the lens used in this study, which is however out of the scope of this paper.

In conclusion, the comparison between results obtained with checkerboard and grid is not performed with the very same contrast in the images. This disadvantages the checkerboard because the contrast and the modulus $K$ are proportional according to Eq. 6 (through the amplitude $A$ of the signal), and the higher the value of $K$, the lower the noise level in the displacement and strain maps according to Eq. 8.

\subsection{Sensor noise propagation}

Fig. 9-a shows the empirical displacement resolutions $\sigma_{u_{x}}$ and $\sigma_{u_{y}}$, and Fig. 9-b the empirical strain resolutions $\sigma_{\varepsilon_{x x}}, \sigma_{\varepsilon_{y y}}$ and $\sigma_{\varepsilon_{x y}}$ as a function of $\rho$. These quantities were measured for a standard deviation of the Gaussian window $\ell$ equal to the period $p^{\prime}$ of the pattern, so that the same spatial resolution was obtained for the two types of patterns (checkerboard and grid), and for all the values of $\rho$ considered here.

Several remarks can be drawn from these curves. First, the noise is lower for the checkerboard than for the grid. This point is developed further in 4.5 below. Second, the results obtained along the $x$ - and $y$-directions are nearly the same, which is logical. Third and as expected, $\sigma_{\varepsilon_{x y}}$ is lower than $\sigma_{\varepsilon_{x x}}$ and $\sigma_{\varepsilon_{y y}}$ (it can be checked that the ratio between $\sigma_{\varepsilon_{x y}}$ and each of the other two is $\sqrt{2}$ ). Finally, all the curves show that both the displacement and the strain resolutions gently decrease as $\rho$ increases, which is consistent with Eq. 8. The question is however to know if not only the trend, but also the model described by these equations is experimentally verified. If it is considered that all the quantities involved in these equations 


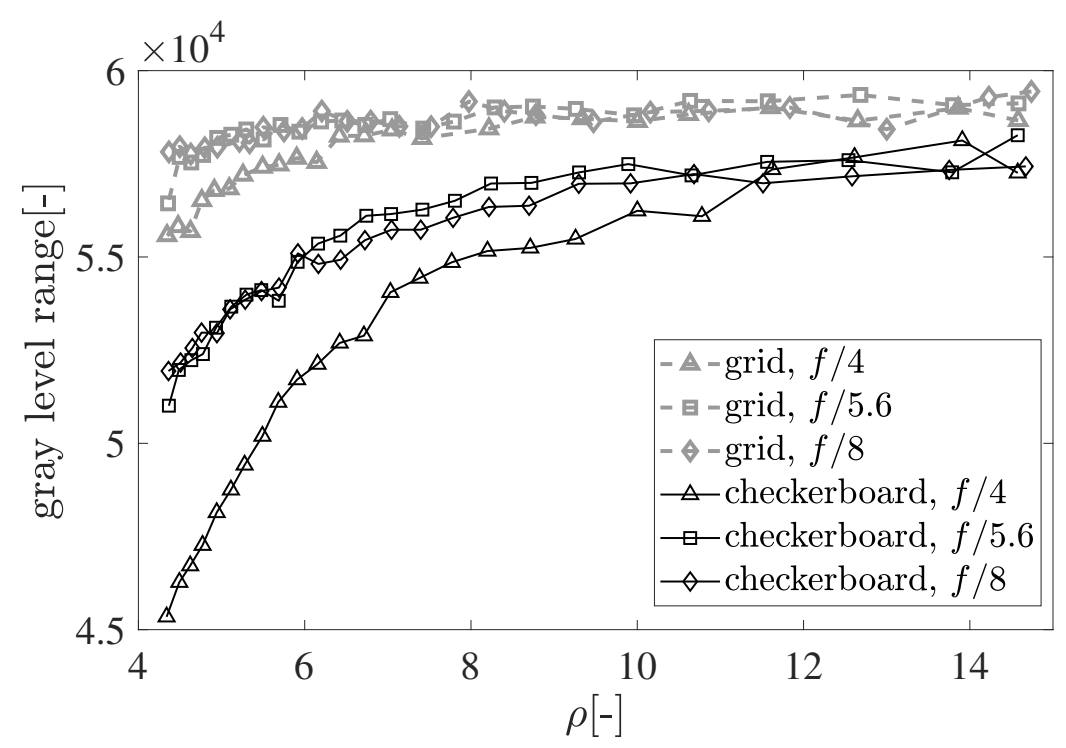

Figure 8: Influence of the aperture on the contrast in the figures.

are constant and do not depend on $\rho$, these resolutions should be inversely proportional to $\rho$. In this case, using a logarithmic scale to plot the results shown in Fig. 9 would lead to decreasing lines of slope -1. This logarithmic scale is used in Fig. 10 and it clearly appears that, as expected, nearly straight lines are obtained. It means that a power law can be used to model noise evolution as a function of $\rho$, so that both the displacement and the strain resolution should be proportional to $\frac{1}{\rho^{n}}$. The slopes $-n$ of the lines in Fig. 10, estimated here with the robustfit function of Matlab, are reported in Table 2. These values change from one case to another but it is clear that all are lower than -1 , which is the value predicted by the model. It means that the displacement and strain resolutions modeled with Eq. 8 are not merely inversely proportional to $\rho$. The reason is probably that some of the quantities involved in these equation actually depends on $\rho$. It is necessary to examine in detail the different terms involved in these equations to find the cause of this phenomenon. Beyond finding why the degree of $\rho$ involved in the predictive formulas is not exactly -1 , it is also necessary to examine if these formulas are experimentally verified. This is the aim of the next section.

\subsection{Prediction of the displacement and strain resolutions}

Determining from the experiments the empirical values of $\sigma_{u_{i}}$ and $\sigma_{\varepsilon_{i j}}$ defined in Eq. 19 makes it possible to check whether their theoretical values given by Eq. 8 are in good agreement. Let us first reexamine the ingredients that feed these latter equations in order to see which one is potentially a function of $\rho$. 


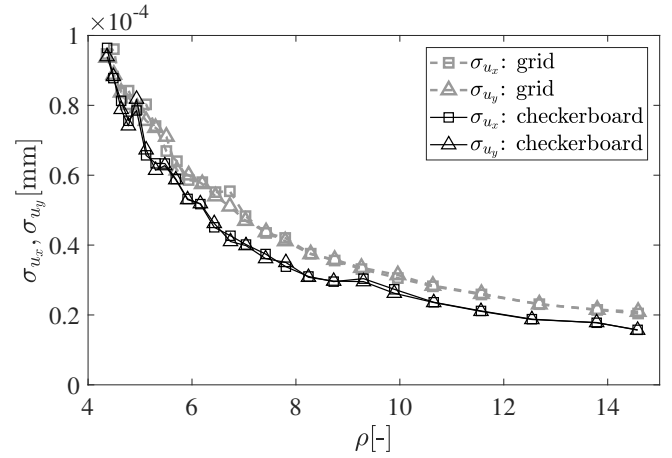

a- Displacement resolutions $\sigma_{u_{x}}$ and $\sigma_{u_{y}}$

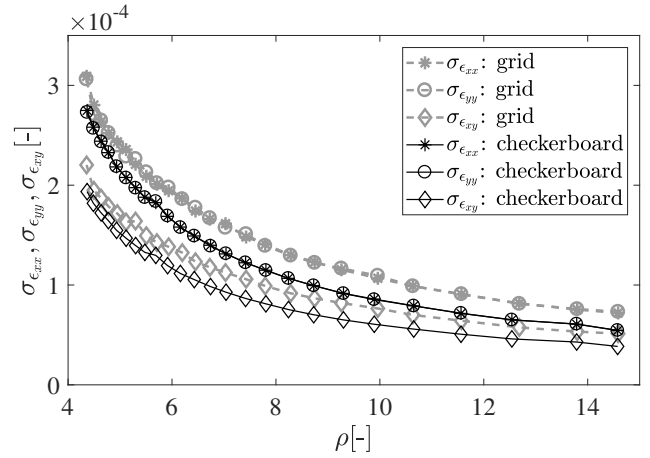

b- Strain resolutions $\sigma_{\varepsilon_{x x}}, \sigma_{\varepsilon_{y y}}$ and $\sigma_{\varepsilon_{x y}}$

Figure 9: Displacement and strain resolutions as a function of $\rho$ for $\ell=p$

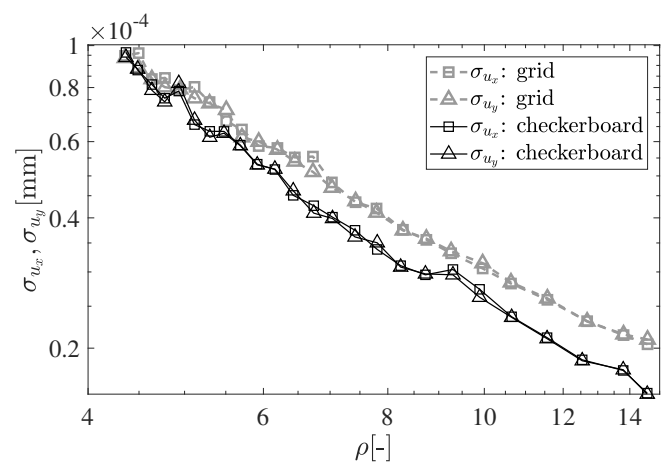

a- Displacement resolutions $\sigma_{u_{x}}$ and $\sigma_{u_{y}}$

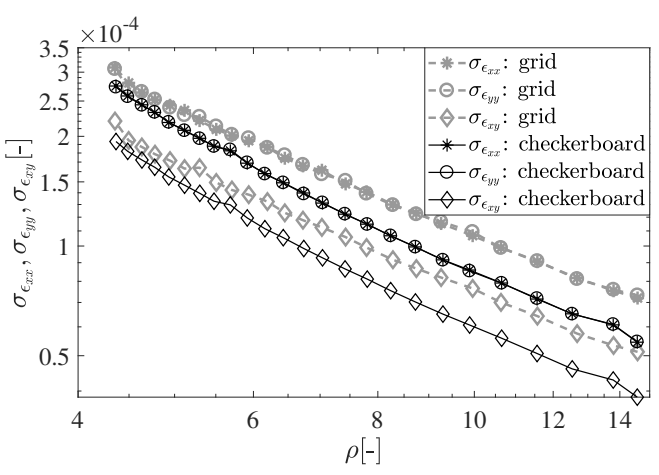

b-Strain resolutions $\sigma_{\varepsilon_{x x}}, \sigma_{\varepsilon_{y y}}$ and $\sigma_{\varepsilon_{x y}}$

Figure 10: Displacement resolutions $\sigma_{u_{x}}$ and $\sigma_{u_{y}}$ as a function of $\rho$, checkerboard pattern. Same experimental results as in Fig. 9 but with a logarithmic scale. 


\begin{tabular}{|c|c|c|}
\hline Pattern & Displacement resolution [m] & Strain resolution [-] \\
\hline Checkerboard & -1.32 & -1.15 \\
\hline Grid & -1.45 & -1.32 \\
\hline
\end{tabular}

Table 2: Slope $-n$ of the curves showing in Fig. 10 the displacement and strain resolutions as a function of $\rho$ with a $\log -\log$ scale.

Quantities depending on $\rho$ The quantities involved in the predictive Eq. 8 are

1. $p^{\prime}$, which is equal here to $0.2 \mathrm{~mm}$ in all cases;

2. $p$, which is equal to $p^{\prime} \times \frac{\sqrt{2}}{2}=0.2 \times \frac{\sqrt{2}}{2} \mathrm{~mm}$ for the checkerboard specimen, and to $p^{\prime}=0.2 \mathrm{~mm}$ for the grid specimen;

3. $\ell$, which is equal to $\ell=p^{\prime}=0.2 \mathrm{~mm}$ in this section for both types of pattern, so that the spatial resolution is the same for both. Other values of $\ell$ are considered in 4.4 below;

4. $\sigma_{\text {image }}$, which is estimated with the value given by Eq. 18;

5. $K$, which is considered here as equal to the mean value of the modulus of the WFT estimated over the rectangular region of dimensions $M \times N$;

6. and $\rho$.

$p, p^{\prime}$ and $\ell$ are therefore constant for all the experimental points on the curves shown above. The question is to see whether $\sigma_{\text {image }}$ and $K$, which are functions of $x$ and $y$ since they both depend of the local brightness, also depend on $\rho$. The answer is given by Fig. 11, which represents these two quantities as a function of $\rho$.

Influence of $\rho$ on $\sigma_{\text {image }}$ Concerning $\sigma_{\text {image }}$ shown in Fig. 11-a, the first remark is that this quantity is lower for the grid. Another point is that for both patterns, $\sigma_{\text {image }}$ gently decreases as $\rho$ increases. These phenomena can be explained by considering the gray level histograms shown in Fig. 12. Indeed, it can be seen that the grid is globally darker than the checkerboard. It can also be observed that the left-hand tail of these gray level distributions is shifted to the left for the highest values of $\rho$. There are two reasons for this. First the effect of the PSF, which can be regarded as an image filtering (and thus as a blurring), becomes lower as $\rho$ increases, which tends to increase the contrast in the images. Since the maximum of the gray level is bounded by $2^{16}-1$ (the aperture and shutter time were adjusted during the experiments so that the right-hand tail just touches this maximum value) the gray level distributions is progressively stretched to the lowest values, thus leading for both types of pattern to a global darkening of the images as $\rho$ increases. Again, since sensor noise is heteroscedastic, the direct consequence is that noise affecting the images $\left(\sigma_{\text {image }}\right)$ globally decreases as $\rho$ increases. 


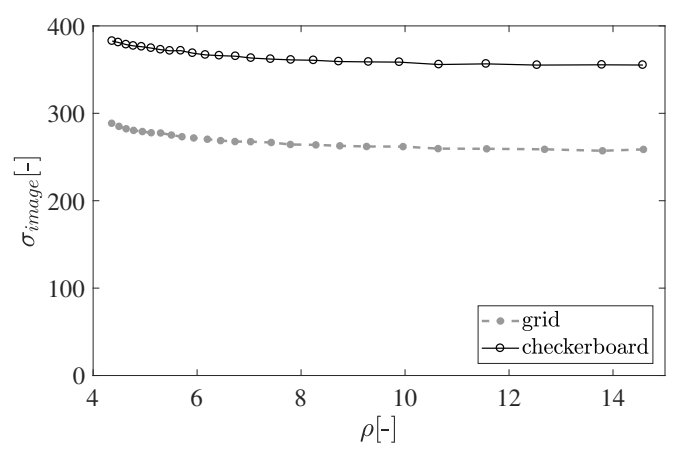

a- $\sigma_{\text {image }}$

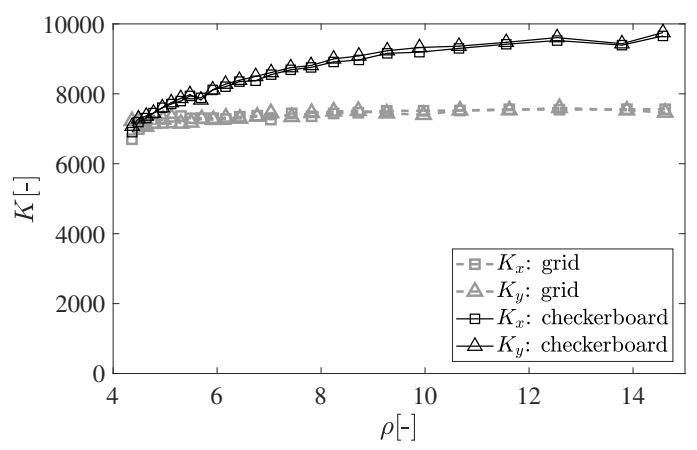

b- $K$

Figure 11: $\sigma_{\text {image }}$ and $K$ as a function of $\rho$.
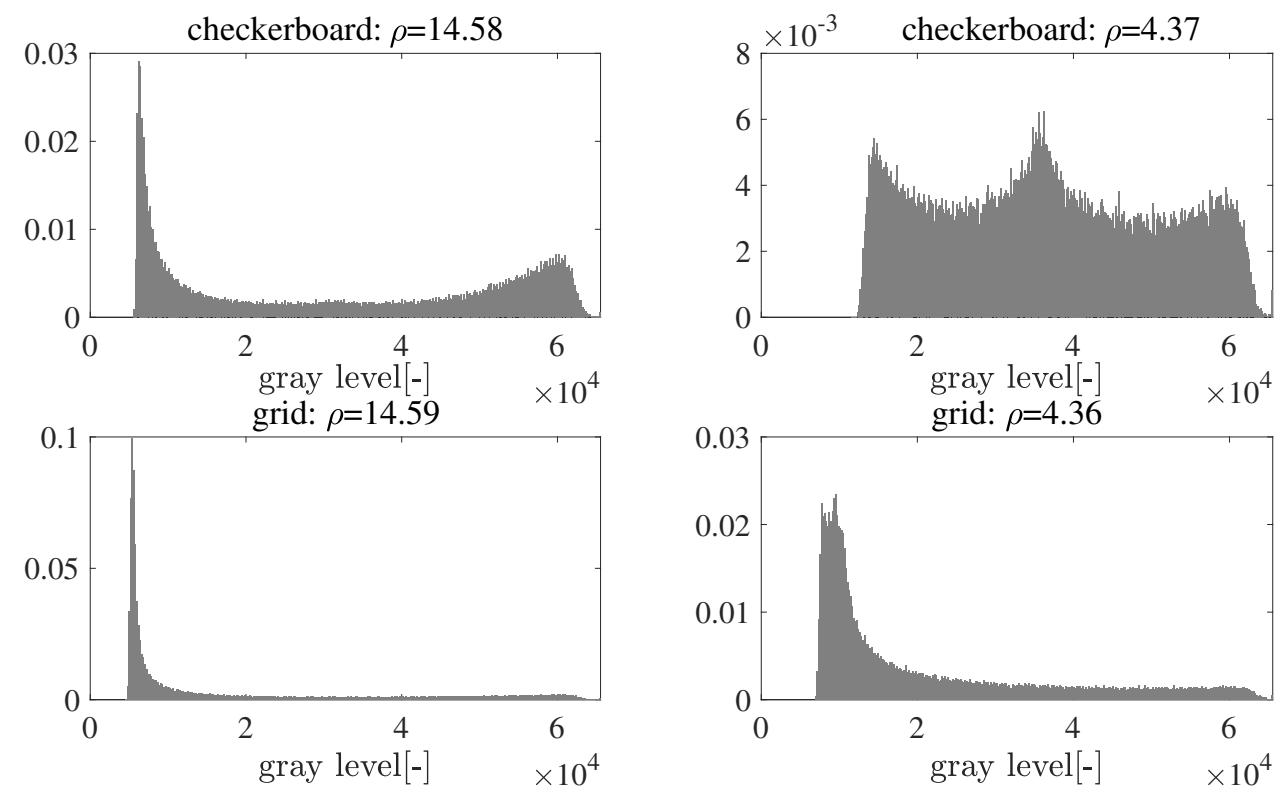

Figure 12: Typical gray level distributions for high and low values of $\rho$

Influence of $\rho$ on $K$ Concerning the modulus of the WFT $K$, it is clear from Fig. 11-b that this quantity increases as $\rho$ increases, which is especially true for the checkerboard, and this increase is non-linear. Eq. 6 shows that $K$ is proportional to the contrast $\gamma$ in the image. As mentioned above, this contrast increases as $\rho$ increases because of a decreasing influence of the PSF, which is especially true for the checkerboard pattern. The point is however that 
according to Eq. $6, K$ is also directly proportional to $d_{1}$, which is the first non-null term in the Fourier development of the periodic pattern profile along the direction for which the WFT is calculated [27]. This quantity reflects the "similarity" of the periodic pattern profile to a sine function along this direction. It is difficult to guess a priori how the product between $d_{1}$ and $\gamma$, which is involved in the definition of $K$ in Eq. 6, evolves as $\rho$ increases, but Fig. 11-b suggests that this product increases with $\rho$.

Another striking point is that $K$ is higher with the checkerboard than for the grid. Interpreting this result is however not easy. Since the contrast in checkerboard images is lower than in grid images, a higher value of $K$ for the checkerboard means that its profile along directions $x^{\prime \prime}$ and $y^{\prime \prime}$ in Fig. 2-a is globally closer to the profile of a pure sine function than the profile of a grid along directions $x^{\prime}$ and $y^{\prime}$ defined in Fig. 2-b.

These evolutions of $K$ and $\sigma_{\text {image }}$ on the noise level both lead to the same property: increasing $\rho$ helps reduce the noise level in the displacement and strain maps. This phenomenon is the same for both the checkerboard and the grid. The main conclusion is therefore that both $K$ and $\sigma_{\text {image }}$ are influenced by $\rho$, and that this influence still leads a power law to be a suitable model to describe the evolution of the displacement and strain resolutions as a function of $\rho$, but with a power lower than -1 , which was difficult to guess a priori.

Processing the images with a window sampled with a constant number of pixels The preceding results were obtained by using in the WFT a Gaussian window featuring the same width since the standard deviation $\ell$ of this Gaussian window was alway equal to the period $p^{\prime}=0.2 \mathrm{~mm}$ of the periodic pattern. This choice for a constant value was justified by the fact that the results, obtained for various numbers of pixels per period $\rho$, could be compared with a spatial resolution being the same in all cases, whatever the value of $\rho$ and whatever the type of pattern: grid or checkerboard. The obtained results show, among others, that the noise level decreases as $\rho$ increases, which is not really surprising, and that the PSF all the more negatively impacts the results as $\rho$ is small. This last phenomenon can also be experimentally evidenced by sampling in all cases the Gaussian window with the same number of pixels in all cases instead of the same value of considering $\ell$ in meter. The benefit is that the results obtained in this case are no longer influenced by the fact that the measurement resolution automatically decreases (thus is improved) merely by averaging, by considering an increasing number of pixels to sample the Gaussian window. In practice however, it is generally not possible to decrease the measurement resolution (thus to improve it) by increasing the number of pixels to encode a given pattern, both the field of view and the sensor size being imposed by experimental conditions. The results above were therefore processed again, but this time with a Gaussian window featuring the same standard deviation in pixel for all values of $\rho$. The spatial resolution (in meter) is not the same from one value of $\rho$ to another since the pixel size $c$ changes, but it is expected that the averaging effect no longer influences the results here.

The influence of $\rho$ can be predicted in this particular case by considering Eq. 7, in which a change of variables in introduced. Indeed, switching from meters to pixels is performed by dividing all lengths $x$ given in meter by the pixel size $c$ expressed in meter, which gives 


\begin{tabular}{|c|c|c|c|}
\hline Pattern & $\begin{array}{c}\text { Displacement } \\
\text { resolution[m] }\end{array}$ & $\begin{array}{c}\text { Displacement } \\
\text { resolution[-] }\end{array}$ & $\begin{array}{c}\text { Strain } \\
\text { resolution }[-]\end{array}$ \\
\hline Checkerboard & $-0.29(\rho>7)$ & $0.71(\rho>7)$ & $0.76(\rho>6)$ \\
\hline Grid & $-0.18(\rho>7)$ & $0.82(\rho>7)$ & $0.87(\rho>6)$ \\
\hline
\end{tabular}

Table 3: Slope $n$ of the linear part of the curves plotted in Fig. 13. The portion of the curves considered to estimate the slope is given in parentheses.

a unitless number denoted by $x_{p x} . p, \ell$ and $c$ are concerned by this change of variables in these equations. Bearing in mind that $\rho$ is precisely the period $p^{\prime}$ expressed in pixel, that $p^{\prime}$ is either equal to $p$ (for the grid) or to $\frac{\sqrt{2}}{2} p$ (for the checkerboard), one can easily check in Eq. 7 after this change of variables that $\sigma_{u}$ is expected not to depend on $\rho$, while $\sigma_{\varepsilon}$ is expected to be a linear function of $\rho$. If $\sigma_{u}$ is also expressed in pixel instead of in meter, then it is expected to be also a linear function of $\rho$, see details in Appendix B.

Fig. 13 shows the displacement and strain resolution estimated with a constant value of $\ell_{p x}$ equal to the maximum value of $\ell_{p x}$ found in this study, namely 14.58 pixels. This value enables us to respect the condition proposed in [26] on the minimum value of the standard deviation of the Gaussian that shall be considered in the WFT, namely that it shall be equal to the period $p^{\prime}$ of the periodic pattern. A log-log scale is used to directly see if a power law can be used to model the observed phenomenon, the slope directly providing the power of this law. It can be seen that the curves obtained for $\sigma_{\varepsilon}$ in Fig. 13-c are in agreement with theoretical expectation, namely that the response is linear with this scale. This is less true when considering the lowest values of $\rho$. The slope is theoretically equal to 1 since $\sigma_{\varepsilon}$ is expected to be a linear function of $\rho$. The slope estimated for these different curves is given in Table 13. It is in fact lower than 1, which confirms the results found just above with a constant value of $\ell$ instead of a constant value of $\ell_{p x}$. Again the reason is that $K$ and $\sigma_{\text {image }}$ are both influenced by contrast, thus by the PSF of the lens, and this effect becomes all the more less marked as $\rho$ increases. The fact that $\sigma_{u}$ should be independent of $\rho$ and that $\sigma_{u_{p x}}$ should linearly depend on $\rho$ is less satisfied for the displacement resolutions showed in Fig. 13-a and -b, in particular for the lowest values of $\rho$. A possible cause is the presence of micro-vibrations, whose effect is not completely removed by using the correction proposed in 3.2. Indeed, derivation strongly diminishes this effect, as discussed for the $\sigma_{\varepsilon}$ curves. However, it is not completely eliminated, which means that another phenomenon occurs for the lowest values of $\rho$, perhaps the fact that the number of pixels per period is too low to correctly encode the sought information. As for the strain resolution and for the same reasons, the slope of the curves estimated over the linear part of the response is lower than 1 and 0 for $\sigma_{u}$ and $\sigma_{u_{p x}}$, respectively. The corresponding values are given in Table 3. 


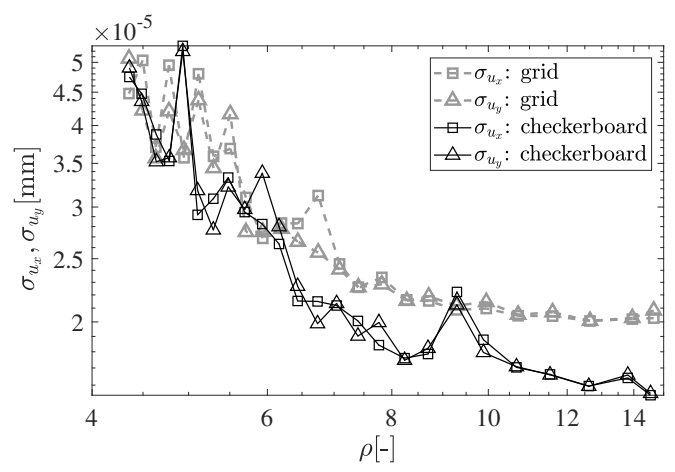

a- Displacement resolution $\sigma_{u}$

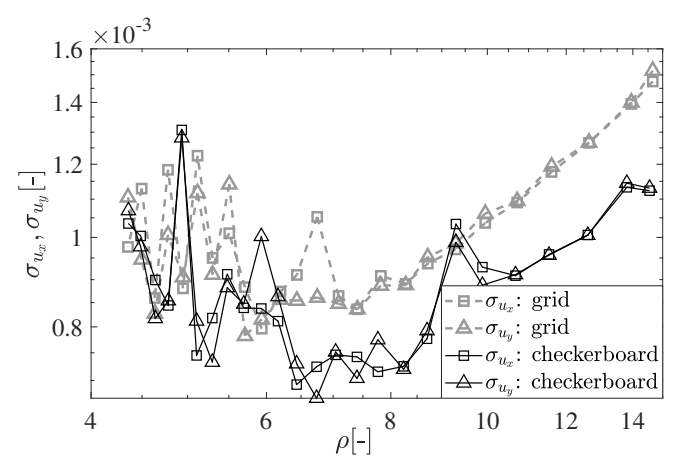

b- Displacement resolution $\sigma_{u_{p x}}$

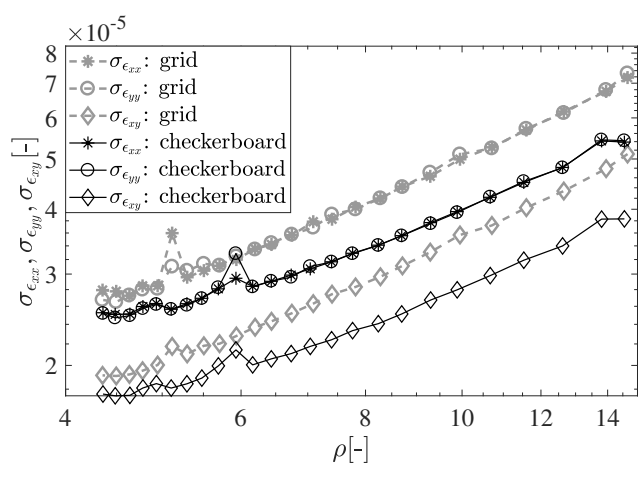

c- Strain resolution

Figure 13: Displacement and strain resolutions as a function of $\rho$ for $\ell_{p x}=14.58$ pixels in all cases, checkerboard and grid patterns.

\subsection{Validation of the predictive formula for the displacement and strain resolutions}

The objective here is to check if the predictive formulas given in Eq. 8 are experimentally verified when they are fed with experimental values of $K$ and $\sigma_{\text {image }}$ estimated with Eq. 17 . Only the checkerboard pattern is considered for the sake of simplicity. Fig. 14 shows the displacement and strain resolutions given by the predictive formulas of Eq. 8. Their empirical counterparts deduced from the experiments through Eq. 19 are superimposed for comparison purposes. The results discussed in the preceding section were obtained with $\ell=p^{\prime}=0.2 \mathrm{~mm}$ only. Two other values are considered here, namely $\ell=1.5 \times p^{\prime}=0.3 \mathrm{~mm}$ and $\ell=2 \times p^{\prime}=$ $0.4 \mathrm{~mm}$, which means that the Gaussian kernel used in the PSF progressively increases from one case to each other.

The experimental results and the values given by the model are in good agreement. Both the displacement and the strain resolutions decrease as $\ell$ increases, which is logical 


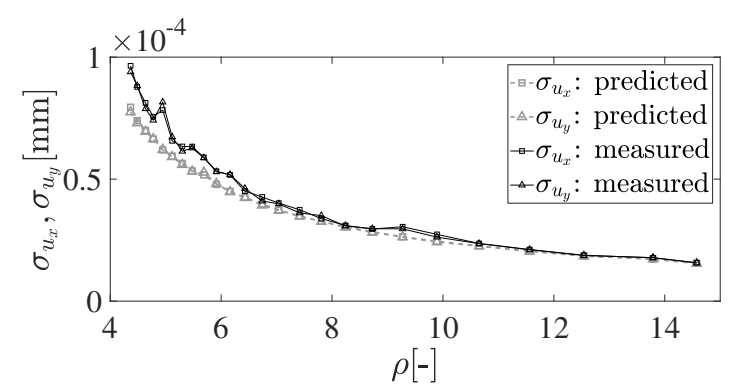

a- $\sigma_{u_{x}}$ and $\sigma_{u_{y}}$ for $\ell=p^{\prime}$

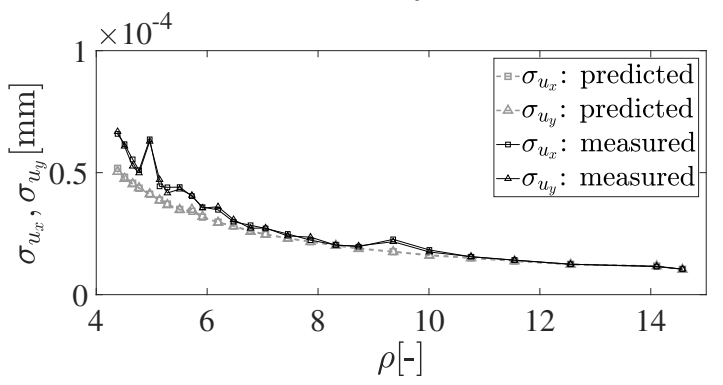

c- $\sigma_{u_{x}}$ and $\sigma_{u_{y}}$ for $\ell=1.5 \times p^{\prime}$

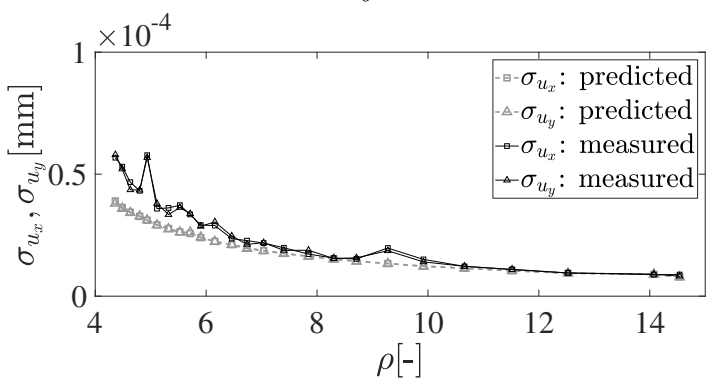

e- $\sigma_{u_{x}}$ and $\sigma_{u_{y}}$ for $\ell=2 \times p$

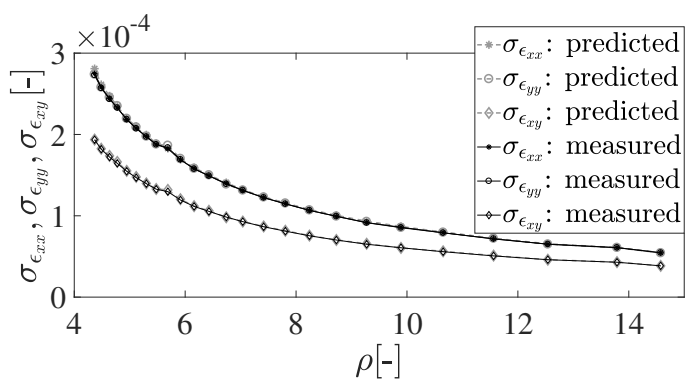

b- $\sigma_{\varepsilon_{x x}}, \sigma_{\varepsilon_{y y}}$ and $\sigma_{\varepsilon_{x y}}$ for $\ell=p$

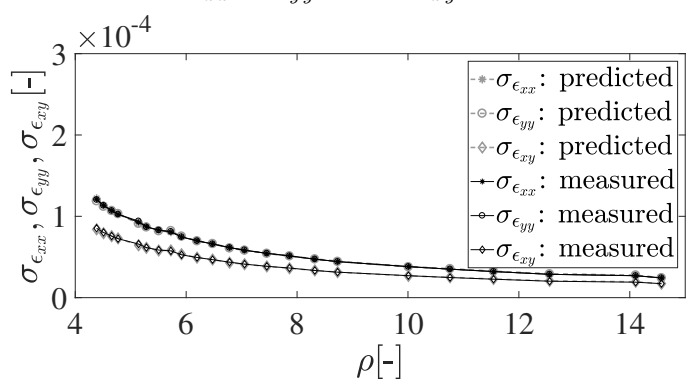

d- $\sigma_{\varepsilon_{x x}}, \sigma_{\varepsilon_{y y}}$ and $\sigma_{\varepsilon_{x y}}$ for $\ell=1.5 \times p$

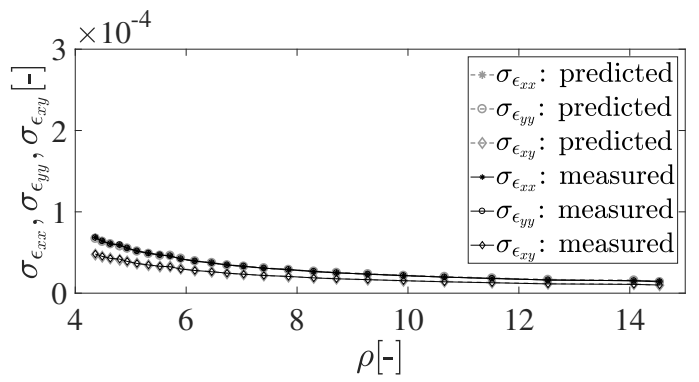

f- $\sigma_{\varepsilon_{x x}}, \sigma_{\varepsilon_{y y}}$ and $\sigma_{\varepsilon_{x y}}$ for $\ell=2 \times p^{\prime}$

Figure 14: Comparison between predicted and measured values of the displacement resolutions $\sigma_{u_{x}}$ and $\sigma_{u_{y}}$ as a function of $\rho$ for three values of $\ell$, checkerboard pattern.

since an increasing number of pixels is taken into account when extracting the phases, thus progressively averaging out the noise. The main difference between model and experiment can be observed for the displacement resolution and for the lowest values of $\rho$. This difference increases as $\ell$ increases. Since this difference mainly concerns the displacement resolution, it is maybe due to the fact that RBM is not correctly removed for these values, and/or that the number of pixels is too low to correctly encode the sought information. 


\subsection{Comparison between checkerboard and grid}

In Fig. 9, an important remark is that both the displacement and strain resolutions are systematically lower for the checkerboard than for the grid, which confirms and generalizes the conclusion given in [19] since the latter was obtained for one value of $\rho$ only. The cause of this difference is given by Eq. 8 and by the preceding remarks on $K$ and $\sigma_{\text {image }}$.

Indeed, the quantities involved in the predictive Eq. 8 are inversely proportional to $\frac{K}{\sigma_{\text {image }}}$, which represents the signal-to-noise ratio (SNR) for the phases. Since these phases are then multiplied (among others) by the period $p$ of the pattern defined in Fig. 2, and since this period is $\sqrt{2}$ times greater for the grid than for the checkerboard, $\frac{K}{\sigma_{\text {image }}}$ shall be multiplied by $\sqrt{2}$ for the checkerboard to fairly compare the SNR between grid and checkerboard in terms of displacement and strain.

It has been seen above that $K$ and $\sigma_{\text {image }}$ evolve in a different way as a function of $\rho$ for each type of pattern, but the fact that the displacement and strain resolutions are always lower for the checkerboard than for the grid is merely due to the fact that $K$ and $\sigma_{\text {image }}$ combine with each other in such a way that the SNR, namely $\frac{K}{\sigma_{\text {image }}}$ for the grid and $\sqrt{2} \times \frac{K}{\sigma_{\text {image }}}$ for the checkerboard, is always greater for the checkerboard than for the grid.

Fig. 15 shows the ratio between the resolution obtained for the checkerboard and its counterpart obtained for the grid. These resolutions are obtained by fitting the experimental results with a power law and dividing the expression found for the checkerboard by its counterpart found for the grid. This ratio is always lower than 1, thus experimentally confirming the fact that checkerboards are better patterns than grids in terms of sensor noise propagation to the final displacement and strain maps. It is worth noting that the difference in performance between the two types of patterns increases as $\rho$ increases, with a maximum relative difference of $20 \%$ for the displacement and $25 \%$ for the strain for the highest values of $\rho$.

\section{Conclusion}

The influence of the sampling density of periodic patterns on sensor noise propagation to displacement and strain maps was studied in this paper. Periodic patterns such as checkerboard and 2D grid were considered, the former being expected to be optimal for measuring displacements and strains in terms of sensor noise propagation. The sampling density was merely estimated by the number of pixels per period $\rho$. The patterns being periodic, the minimization of the optical residual was not performed in the spatial domain with Digital Image Correlation but in the Fourier domain, with as spectral method, namely the Localized Spectrum Analysis. Theoretical predictions claim that the noise level in these maps, which was estimated here through the displacement and strain resolutions calculated with a constant spatial resolution from one case to another, is inversely proportional to $\rho$. The main 


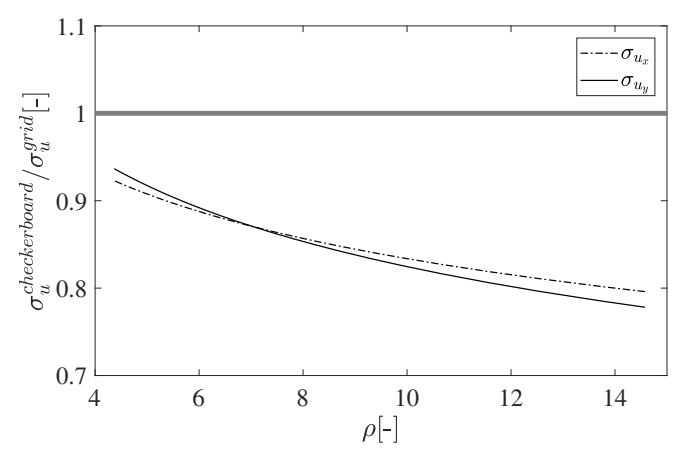

a- Ratio between displacement resolution for the checkerboard and the grid

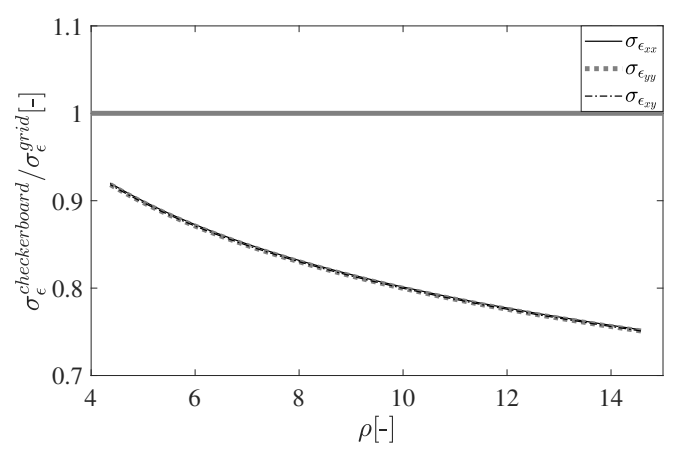

b- Ratio between strain resolution for the checkerboard and the grid

Figure 15: Ratio between displacement and strain resolutions for the checkerboard and the grid.

conclusion is that this is not exactly the case, some quantities such as the modulus of the Windowed Fourier Transform and the mean value of the noise in the images themselves being also impacted by $\rho$. A conclusion of this study is that the influence of $\rho$ on the displacement and strain resolutions can reasonably be modeled with a power law, as claimed by theoretical predictions, but with a power lower than -1. Another conclusion is that it is confirmed that checkerboards lead to a lower noise level in displacement and strain maps than $2 \mathrm{D}$ grids, the difference between the two increasing as $\rho$ increases. It has been observed during these experiments that the contrast in checkerboard images was lower than the contrast in $2 \mathrm{D}$ grids whatever the value of $\rho$. This effect is presumably due the Point Spread Function of the lens. Other lenses featuring a better resolution power than the one of the lens used in the present study should be considered in future studies to confirm this point, and to give even lower noise levels in displacement and strain maps than the ones characterized in the present work.

Acknowlegements. The authors gratefully acknowledge the financial support of the Beijing Institute of Technology, China, for funding the stay in France of S. Qin. 


\section{Appendix A: definition of the metrological parameters used in this study}

Different metrological parameters are discussed in this paper, namely the measurement resolution, the bias and the spatial resolution. The measurement resolution can either be the displacement resolution or the strain resolution. All these quantities are throughly defined in [8]. These definitions are recalled below for the sake of completeness and clarity:

Measurement resolution: in Ref. [46], the measurement resolution is defined by the smallest change in a quantity being measured that causes a perceptible change in the corresponding indication. More precisely, it is proposed in [47] to define it as the change in quantity being measured that causes a change in the corresponding indication greater than one standard deviation of the measurement noise, which enables us to quantify the measurement resolution. This definition is quite arbitrary, any other (reasonable) multiple of the standard deviation being also potentially acceptable, but the idea is that the resolution quantifies the smallest change not likely to be caused by measurement noise [47]. The measurement resolution is here either the displacement resolution or the strain resolution, depending on the quantity of interest.

Bias: there are several causes for the systematic error observed with full-field measurement techniques. We consider here the so-called matching bias, which concerns both DIC and LSA. A classic way to assess it is to consider a synthetic reference sine function with a given amplitude, and to consider that the relative loss of amplitude quantifies this bias, as in Ref. [48, 49, 50] for DIC or in [51, 52] for LSA. The quantity can be estimated by considering the displacement field returned by a given technique when processing a pair of synthetic images, the current one being the reference one deformed through a particular wave defined in $[52,29]$ The bias is denoted by $\lambda$. The systematic error due to the interpolation function used to have both the reference and the deformed images in the same coordinate system [53, 23, 54] is not considered here because it concerns only DIC and not LSA [29].

Spatial resolution: the spatial resolution denoted by $\ell$ is defined here by the lowest period of a sinusoidal deformation that the technique is able to reproduce before losing a certain percentage of amplitude, in other words before the bias reaches a certain value, this quantity being chosen a priori [49]. The advantage of this definition is that it is not based on an arbitrary value for the subset size in DIC or for the window used while processing a periodic pattern with LSA. This makes it possible to compare the spatial resolution between these two techniques, whose principle is totally different. This definition of the spatial resolution holds here for the phase, and consequently for the displacement. It also holds for the phase derivatives and the strain components if no smoothing is performed before differentiating the phases and the displacements. Otherwise the spatial resolution is all the more impaired as the width of the filter increases. 


\section{Appendix B: influence of $\rho$ on the displacement and strain res- olutions with a width of the Gaussian window constant in pixel}

In the following, symbol $\sim$ means "proportional to". $K$ and $\sigma_{\text {image }}$ being assumed to be constant, one can deduce from Equation 7:

$$
\left\{\begin{aligned}
\sigma_{u}[m] & \sim \frac{p c}{\ell} \\
\sigma_{\varepsilon}[-] & \sim \frac{p c}{\ell^{2}}
\end{aligned}\right.
$$

Let now give any length in pixel instead of in meter in the expressions of $\sigma_{u}$ and $\sigma_{\varepsilon}$ above. Denoting by $x_{p x}$ any length expressed in pixel, $x$ being its counterpart expressed in meter, we have $x=x_{p x} \times c$, where $c$ is the size in meter of the square region covered by a pixel on the specimen. We can deduce from Equation 20

$$
\left\{\begin{aligned}
\sigma_{u}[m] & \sim \frac{p_{p x} c}{\ell_{p x}} \\
\sigma_{\varepsilon}[-] & \sim \frac{p_{p x}}{\ell_{p x}^{2}}
\end{aligned}\right.
$$

Assuming now the Gaussian window used in the WFT has the same width in all cases, $\ell_{p x}$ is constant. Thus, we have in this particular case

$$
\left\{\begin{aligned}
\sigma_{u}[m] & \sim p_{p x} c \\
\sigma_{\varepsilon}[-] & \sim p_{p x}
\end{aligned}\right.
$$

We can also express $\sigma_{u}$ in pixel instead of in meter. We have, as for any length, $\sigma_{u_{p x}}=\frac{\sigma_{u}}{c}$. Thus

$$
\left\{\begin{aligned}
\sigma_{u}[m] & \sim p_{p x} c \\
\sigma_{u_{p x}}[-] & \sim p_{p x} \\
\sigma_{\varepsilon}[-] & \sim p_{p x}
\end{aligned}\right.
$$

Bearing in mind that $\rho$ is the number of pixels per period $p^{\prime}$ (see the definition of $p^{\prime}$ in Fig. 2 above), we have $p_{p x}=\frac{\sqrt{2}}{2} \rho$ for the checkerboard and $p_{p x}=\rho$ for the grid. Hence, we can deduce from Equation 23: 


$$
\begin{cases}\sigma_{u}[m] & \sim \rho c \\ \sigma_{u_{p x}}[-] & \sim \rho \\ \sigma_{\varepsilon}[-] & \sim \rho\end{cases}
$$

$c$ is the size of a pixel in meter, thus $c=\frac{p^{\prime}}{\rho}$ and

$$
\begin{cases}\sigma_{u}[m] & \text { does not depend on } \rho \\ \sigma_{u_{p x}}[-] & \sim \rho \\ \sigma_{\varepsilon}[-] & \sim \rho\end{cases}
$$




\section{References}

[1] P. L. Reu, B. Blaysat, J. Helm, E. M.C. Jones, and M. Iadicola. Update on the DIC challenge 2.0 and the stereo-DIC challenge. In SEM annual conference "Expanding the Boundaries of Mechanics", Reno, USA, 2019. Proceedings of the conference.

[2] D. Dang, R. Moutou-Pitti, E. Toussaint, and M. Grédiac. Investigating wood under thermo-hydromechanical loading at the ring scale using full-field measurements. Wood Science \& Technology, 52(6):1473-1493, 2018.

[3] I. Khlifi, O. Pop, J.-C. Dupr, P. Doumalin, and M. Huger. Investigation of microstructure-property relantionships of magnesia-hercynite refractory composites by a refined digital image correlation technique. Journal of the European Ceramic Society, 39(13):3893-3902, 2019.

[4] F. Mathieu, F. Hild, and S. Roux. Identification of a crack propagation law by digital image correlation. International Journal of Fatigue, 36(1):146-154, 2012.

[5] R. Moutou Pitti, C. Badulescu, and M. Grédiac. Characterization of a cracked specimen with full-field measurements: direct determination of the crack tip and energy release rate calculation. International Journal of Fracture, 187(1):109-121, 2014.

[6] M. Grédiac and F. Hild, editors. Full-field measurements and identification in solid mechanics. Wiley, 2012. ISBN: 9781848212947, 496 pages.

[7] F. Pierron and M. Grédiac. Towards material testing 2.0. a review of test design for identification of constitutive parameters from full-field measurements. Strain, 2020. Accepted, in press.

[8] M. Grédiac, B. Blaysat, and F. Sur. A critical comparison of some metrological parameters characterizing local digital image correlation and grid method. Experimental Mechanics, 57(3):871-903, 2017.

[9] M. Sutton, J.J. Orteu, and H. Schreier. Image Correlation for Shape, Motion and Deformation Measurements. Basic Concepts, Theory and Applications. Springer, 2009.

[10] D. Lecompte, A. Smits, S. Bossuyt, H. Sol, J. Vantomme, D. Van Hemelrijck, and A.M. Habraken. Quality assessment of speckle patterns for digital image correlation. Optics and Lasers in Engineering, 44(11):1132-1145, 2006.

[11] G. Stoilov, V. Kavardzhikov, and D. Pashkouleva. A comparative study of random patterns for digital image correlation. Journal of theoretical and Applied Mechanics, 42(2):55-66, 2012.

[12] S. Bossuyt. Optimized patterns for digital image correlation. In Proceedings of the 2012 Annual Conference on Experimental and Applied Mechanics, Volume 3: Imaging Methods for Novel Materials and Challenging Applications, 2013. 
[13] G.F. Bomarito, J.D. Hochhalter, T.J. Ruggles, and A.H. Cannon. Increasing accuracy and precision of digital image correlation through pattern optimization. Optics and Lasers in Engineering, 91:73 - 85, 2017.

[14] G.F. Bomarito, J.D. Hochhalter, and T.J. Ruggles. Development of optimal multiscale patterns for digital image correlation via local grayscale variation. Experimental Mechanics, 58(7):11691180, 2018. Accepted, online.

[15] X. Xu, X. Ren, F. Zhong, C. Quan, and X. He. Optimization of speckle pattern based on integer programming method. Optics and Lasers in Engineering, 133:106100, 2020.

[16] J. Réthoré. A fully integrated noise robust strategy for the identification of constitutive laws from digital images. Internation Journal for Numerical Methods in Engineering, 84(6):631-660, 2010.

[17] B. Pan, Z. Lu, and H. Xie. Mean intensity gradient: an effective global parameter for quality assessment of the speckle patterns used in digital image correlation. Optics and Lasers in Engineering, 48(4):469-77, 2010.

[18] M. Grédiac, B. Blaysat, and F. Sur. On the optimal pattern for displacement field measurement: random speckle and DIC, or checkerboard and LSA? Experimental Mechanics, 60(4):509534, 2020.

[19] M. Grédiac, B. Blaysat, and F. Sur. Extracting displacement and strain fields from checkerboard images with the localized spectrum analysis. Experimental Mechanics, 59(2):207-218, 2019.

[20] S. Fayad and P. L. Reu. Pattern induced bias in out-of-plane motion in digital image correlation. In SEM annual conference "Expanding the Boundaries of Mechanics", Reno, USA, 2019. Proceedings of the conference.

[21] S. S. Fayad, D. T. Seidl, and P. L. Reu. Spatial DIC errors due to pattern-induced bias and grey level discretization. Experimental Mechanics, 60(2):249-263, 2020.

[22] F. Sur, B. Blaysat, and M. Grédiac. On biases in displacement estimation for image registration, with a focus on photomechanics. 2020. Submitted.

[23] Y. Su, Q. Zhang, Z. Gao, X. Xu, and X. Wu. Fourier-based interpolation bias prediction in digital image correlation. Optics Express, 23(15):19242-19260, 2015.

[24] M. Grédiac, F. Sur, and B. Blaysat. Comparing several spectral methods used to extract displacement and strain fields from checkerboard images. Optics and Lasers in Engineering, 127:105984, 2020.

[25] P. Reu. All about speckles: Aliasing. Experimental Techniques, 38(5):1-3, 2014.

[26] F. Sur and M. Grédiac. Influence of the analysis window on the metrological performance of the grid method. Journal of Mathematical Imaging and Vision, 56(3):472-498, 2016. 
[27] F. Sur and M. Grédiac. Towards deconvolution to enhance the grid method for in-plane strain measurement. Inverse Problems and Imaging, 8(1):259-291, 2014.

[28] M. Grédiac and F. Sur. Effect of sensor noise on the resolution and spatial resolution of the displacement and strain maps obtained with the grid method. Strain, 50(1):1-27, 2014. Paper invited for the 50th anniversary of the journal. Wiley.

[29] M. Grédiac, F. Sur, and B. Blaysat. The grid method for in-plane displacement and strain measurement: a review and analysis. Strain, 52(3):205-243, 2016.

[30] E. W. Grafarend. Linear and Nonlinear Models: Fixed Effects, Random Effects, and Mixed Models. Walter de Gruyter, 2006.

[31] F. Sur, B. Blaysat, and M. Grédiac. Determining displacement and strain maps immune from aliasing effect with the grid method. Optics and Lasers in Engineering, 86:317-328, 2016 .

[32] X. Dai, H. Xie, H. Wang, C. Li, Z. Liu, and L. Wu. The geometric phase analysis method based on the local high resolution discrete fourier transform for deformation measurement. Measurement Science and Technology, 25(2):025402, 2014.

[33] X. Dai, H. Xie, and H. Wang. Geometric phase analysis based on the windowed fourier transform for the deformation field measurement. Optics and Laser Technology, 58(6):119-127, 2014.

[34] M. Grédiac, B. Blaysat, and F. Sur. A robust-to-noise deconvolution algorithm to enhance displacement and strain maps obtained by local DIC and LSA. Experimental Mechanics, 59(2):219-243, 2019.

[35] A. Foi, M. Trimeche, V. Katkovnik, and K. Egiazarian. Practical Poissonian-Gaussian noise modeling and fitting for single-image raw-data. IEEE Transactions on Image Processing, 17(10):1737-1754, 2008.

[36] F. Sur and M. Grédiac. Measuring the noise of digital imaging sensors by stacking raw images affected by vibrations and illumination flickering. SIAM Journal on Imaging Sciences, 8(1):611-643, 2015. Society for Industrial and Applied Mathematics.

[37] J.L. Piro and M. Grédiac. Producing and transferring low-spatial-frequency grids for measuring displacement fields with moiré and grid methods. Experimental Techniques, 28(4):23-26, 2004.

[38] R. Hartley and A. Zisserman. Multiple view geometry in computer vision. Cambridge University Press, 2003.

[39] J.-M. Morel and G. Yu. ASIFT: A new framework for fully affine invariant image comparison. SIAM Journal on Imaging Sciences, 2(2):438-469, 2009. 
[40] A.C. Aitken. On least squares and linear combination of observations. Proceedings of the Royal Society of Edinburgh, 55:42-48, 1936.

[41] Sensicam QE - 1288 datasheet, 2005. PCO Imaging Tech. Rep.

[42] F. Sur and M. Grédiac. Sensor noise modeling by stacking pseudo-periodic grid images affected by vibrations. IEEE Signal Processing Letters, 21(4):432-436, 2014.

[43] F. Murtagh, J-L Starck, and A. Bijaoui. Image restoration with noise suppression using a multiresolution support. Astronomy and Astrophysics Supplement Series, 112:179-189, 1995.

[44] F.J. Anscombe. The transformation of Poisson, binomial and negative-binomial data. Biometrika, 35(3-4):246-254, 1948.

[45] B. OShea. Diffraction and detector considerations for high resolution imaging. 2018. SLAC Technical Note: SLAC-TN-18-001. Stanford University.

[46] International vocabulary of metrology. Basic and general concepts and associated terms, 2008. Third edition.

[47] A. Chrysochoos and Y. Surrel. Chapter 1. Basics of metrology and introduction to techniques. In M. Grédiac and F. Hild, editors, Full-field measurements and identification in solid mechanics, pages 1-29. Wiley, 2012.

[48] M. Bornert, F. Brémand, P. Doumalin, J.-C. Dupré, M. Fazzini, M. Grédiac, F. Hild, S. Mistou, J. Molimard, J.-J. Orteu, L. Robert, Y. Surrel, P. Vacher, and B. Wattrisse. Assessment of digital image correlation measurement errors: methodology and results. Experimental Mechanics, 49(3):353-370, 2009.

[49] L. Wittevrongel, P. Lava, S. V. Lomov, and D. Debruyne. A self adaptive global digital image correlation algorithm. Experimental Mechanics, 55(2):361-378, 2015.

[50] J. Blaber, B. Adair, and A. Antoniou. Ncorr: Open-source 2d digital image correlation matlab software. Experimental Mechanics, 2015. doi: 10.1007/s11340-015-0009-1.

[51] C. Badulescu, M. Bornert, J.-C. Dupr, S. Equis, M. Grédiac, J. Molimard, P. Picart, R. Rotinat, and V. Valle. Demodulation of spatial carrier images: Performance analysis of several algorithms. Experimental Mechanics, 53(8):1357-1370, 2013.

[52] M. Grédiac, F. Sur, C. Badulescu, and J.-D. Mathias. Using deconvolution to improve the metrological performance of the grid method. Optics and Lasers in Engineering, 51(6):716-734, 2013.

[53] Y. Q. Wang, M. Sutton, H. Bruck, and H. W. Schreier. Quantitative error assessment in pattern matching: effects of intensity pattern noise, interpolation, strain and image contrast on motion measurements. Strain, 45(2):160 -178, 2009. 
[54] Y. Su, Q. Zhang, X. Xu, and Z. Gao. Quality assessment of speckle patterns for DIC by consideration of both systematic errors and random errors. Optics and Lasers in Engineering, 86:132-142, 2016. 